\title{
An approach to MCGDM based on multi-granulation Pythagorean fuzzy rough set over two universes and its application to medical decision problem
}

\author{
Bingzhen Sun ${ }^{1} \mathbb{D} \cdot$ Sirong Tong ${ }^{1} \cdot$ Weimin $\mathrm{Ma}^{2} \cdot$ Ting Wang $^{1} \cdot$ Chao Jiang $^{3}$
}

Published online: 6 August 2021

(c) The Author(s), under exclusive licence to Springer Nature B.V. 2021

\begin{abstract}
Exploring efficiency approaches to solve the problems of decision making under uncertainty is a mainstream direction. This article explores the rough approximation of the uncertainty information with Pythagorean fuzzy information on multi-granularity space over two universes combined with grey relational analysis. Based on grey relational analysis, we present a new approach to calculate the relative degree or the attribute weight with Pythagorean fuzzy set and give a new descriptions for membership degree and nonmembership. Then, this paper proposes a multi-granulation rough sets combined with Pythagorean fuzzy set, including optimistic multi-granulation Pythagorean fuzzy rough set, pessimistic multi-granulation Pythagorean fuzzy rough set and variable precision Pythagorean fuzzy rough set. Several basic properties for the established models are investigated in detail. Meanwhile, we present an approach to solving the multiple-criteria group decision making problems with fuzzy information based on the proposed model. Eventually, a case study of psychological evaluation of health care workers in COVID-19 show the principle of the established model and is utilized to verify the availability. The main contributions have three aspects. The first contribution of an approach of calculating the attribute weight is presented based on Grey Relational Analysis and gives a new perspective for the Pythagorean fuzzy set. Then, this paper proposes a mutli-granulation rough set model with Pythagorean fuzzy set over two universes. Finally, we apply the proposed model to solving the psychological evaluation problems.
\end{abstract}

Keywords Multigranulation rough set · Pythagorean fuzzy set · Grey relational analysis · Group decision making $\cdot$ Psychological evaluation

Weimin Ma

mawm@tongji.edu.cn

Chao Jiang

jiangbeyound0818@163.com

Extended author information available on the last page of the article 


\section{Introduction}

Decision making is a process that individuals select optimal alternatives from candidates, which usually permeates every aspect of life. As the environment we face become more complicated and changeable, decision making based on only one person is no longer a good response to changing circumstances and group decision making developed. The group decision making aim at obtaining optimal alternatives combined with expert opinions from various fields. The members from group need to have a wide and adequate discussion with respect to decision-making issues and reach a certain consensus through negotiations. In recent years, several new methodologies and decision theories for group decision making under uncertain circumstances are built by many scholars (Sun et al. 2018; Zhang 2016; Faizi et al. 2020; Jiang et al. 2020; Li et al. 2020; Liu and Liu 2020; Ma et al. 2020; Bashir et al. 2020; Sun and Ma 2015), including consensus reaching process (Li et al. 2019; Zhang et al. 2020, 2021b; Bashir et al. 2020; Sun and Ma 2015), information integration and ranking (Sun et al. 2018; Zhang 2016; Jiang et al. 2020; Li et al. 2020; Liu and Liu 2020; Ma et al. 2020), two-sided matching decision making problems(Zhang et al. 2021a) and applications (Faizi et al. 2020).

In many situations for uncertain decision-making problems, Pythagorean fuzzy sets (PFSs), proposed by Yager $(2014 ; 2013)$, are more powerful to character uncertainty and inaccuracy of available information compared to the fuzzy set (Zadeh 1965), intuitionistic fuzzy set (Lang et al. 2019), vague set and interval number, and etc. PFS is an extension of the interval fuzzy set theory by relaxing the condition of the relation of membership and non-membership, where the sum of membership degree and non-membership degree may be bigger than 1, but their square sum is less than or equal 1. PFS has many applications in the researches (Chen 2018; Peng and Dai 2017; Peng and Yang 2015; Garg 2017; Yager 2004). Although many researches emerged for various multi-criteria decision-making problems from different perspectives, there still exist less research to discuss the management meaning of correlative degree by the Pythagorean fuzzy set. Accordingly, this paper utilizes the correlative degree and non-correlative degree to describe the membership degree and non-membership degree. Therefore, this paper improved the grey relative analysis and propose a new GRA model from two opposite directions by considering the correlative degree and non-correlative degree.

As a result of the complexity of the environment in decision making, more and more attention is paid to the application of mathematics in decision-making process. As new emerging mathematical theories develops, the Pawlak rough set theory was proposed to study and analyze various of data from different fields (Pawlak 1982), which has been applied to studying intelligent decision systems with sufficient and incomplete information. The core concepts of rough set theory are equivalent relation and approximation based on upper and lower approximate space. Pawlak rough set has two directions, which include approximation space and reduction of the number of attributes set. Furthermore, the rough set has three conditions (Qian et al. 2010a): (1) The any two criteria need to be satisfied with mutual independence; (2) An intersection operation with regards to two attributes can be satisfied. (3) The target concept is approximately described by utilizing the subset classes which are composed of the combination of two criteria on the universes. Nevertheless, the original rough set exists drawbacks which is not satisfied with the decision making with regards as management science in uncertainty circumstance. On the one hand, single granulation of the universe cannot be contented with the realistic situation. On the other hand, the binary relation provides an perspective to solve the decision making problems 
under uncertain circumstances and refrains from the limitations of the existing models. Based on these problems, a lot of studies have been performed abroad. Qian et al. (2010b) proposed the multi-granulation rough set model to compensate for the drawbacks in the original rough set.

Furthermore, there are many researches in regards as multi-granulation rough set. Multi-granulation rough set becomes a hot issue in the field of management science and appeals to many scholars having various professional backgrounds, including the extension of theories (Huang et al. 2020; Liang et al. 2020; Shao et al. 2020), attribute reduction (Kong et al. 2019; Sun et al. 2020) and the applications in the decision-making problems (Pang et al. 2020; Ali et al. 2018). Accordingly, Qian et al. (2010b) proposed the optimistic multi-granulation rough set and pessimistic multi-granulation rough set derived from the concepts of multiple binary relations on the universe considering the risk attitudes. Huang et al. (2014) conducted intuitionistic fuzzy multi-granulation rough set combing intuitionistic fuzzy set with multi-granulation rough set. In recent years, many scholars have efforts to pay attention to their applications in the fields of management science. Ali et al. (2018) proposed a new conflict decision-making model combined with multi-granulation rough set. Pang et al. (2020) presented a multi-criteria group decision making model based on three-way decision making and multi-granulation rough set. As the uncertainty of decision-making situation increased, the crisp number is no longer satisfied with the circumstance and Pythagorean fuzzy set emerges. In this paper, we investigate a new application in multi-criteria group decision making problems of the multi-granulation rough set with Pythagorean fuzzy set under the framework of the two universes and give the membership and non-membership a new explanation for Pythagorean fuzzy set. For example, the psychological evaluation of health care workers in COVID-19 plays a significant role on the epidemic situation. The process of data analysis consists of many aspects, including feature selection, information integration and conclusion analysis. There are many emotions to describe the psychological situation for the health workers and these emotions have great influence on fighting the epidemic. Sometimes, the information given by the health workers is obscure and need to transfer into fuzzy information to handle it. Therefore, it is necessary for uncertainty decision-making problem to apply multi-granulation rough set over two universes considering the various psychological situations. We describe the psychological situation from various perspectives. Nevertheless, many researches consider optimistic and pessimistic multi-granulation rough set instead of generalized Pythagorean fuzzy multi-granulation rough set and less literatures explored the application to the problem of psychological evaluation. In this paper, the process of decision making including three stages, including weight calculation, information fusion and conclusion analysis for the problem of psychological evaluation. In the stage of weight calculation, we extract the important criteria by using neighborhood rough set-based attribute reduction and describe the relation between the criteria and decision objects combined with grey relational analysis and Pythagorean fuzzy set. In the second stage, the multi-granulation Pythagorean fuzzy set is used to integrate various information. Finally, a case study of psychological evaluation is applied to verify the effectiveness and validity compared with other multicriteria decision making, such as TOPSIS, VIKOR and PROMETHEE (II).

The remain introductions are organized as follows. Sect. 2 restates several basic theories of the theory of rough set theory, Pawlak rough set and the concept of fuzzy set as well as Pythagorean fuuzy set. Section 3 gives the definition of the grey relational analysis based on Pythagorean fuzzy set. Section 4 proposes the model of multi-granulation Pythagorean fuzzy rough set. Subsequently, we details investigate the properties of the multi-granulation Pythagorean fuzzy rough set lower and upper approximation operators established in 
this section. Section 5 introduces an approach to multiple attribute group decision making based on multi-granulation Pythagorean fuzzy rough set over two universes proposed by Sect. 4 and applies it to psychological evaluation for health care workers in COVID-19. A case study describes the availability and validity of the proposed methodology compared other multi-criteria decision making method, including TOPSIS, VIKOR and PROMETHEE (II). Finally, Sect. 6 concludes the research and put out further study directions.

\section{Preliminaries}

In this section, some concepts will be presented, including Pythagorean fuzzy set and their measures, rough set and multi-granulation rough set.

\subsection{Fuzzy set and Pythagorean fuzzy set}

The concepts of fuzzy set and Pythagorean fuzzy set is described in the following part.

Definition 2.1 (Lang et al. 2019; Zadeh 1965) Let $X$ be a non-empty universe. A fuzzy set $A$ with respect to $X$ is represented by $\mu_{A}: X \rightarrow[0,1]$, which $\mu_{A}(x)$ denotes membership function of the element $x(x \in X)$ in $A$. If $X$ is a infinite set, then $A \in F(X)$ could be described as follows:

$$
A=\frac{A\left(x_{1}\right)}{x_{1}}+\frac{A\left(x_{2}\right)}{x_{2}}+\cdots+\frac{A\left(x_{n}\right)}{x_{n}}=\sum_{i=1}^{n} \frac{A\left(x_{i}\right)}{x_{i}} .
$$

Next, we introduce the concept of Pythagorean fuzzy set.

Definition 2.2 A Pythagorean fuzzy set (Yager 2014; Yager and Abbasov 2013) in the universe can be defined as:

$$
P=\left\{\left\langle x, \mu_{P}(x), v_{P}(x)\right\rangle \mid x \in X\right\},
$$

where $\mu_{P}(x) \in[0,1]$ denotes the degree of membership and $v_{P}(x) \in[0,1]$ denotes the degree of non-membership with condition:

$$
0 \leq\left(u_{P}(x)\right)^{2}+\left(v_{P}(x)\right)^{2} \leq 1 .
$$

Let $P=\left[u_{P}(x), v_{P}(x)\right]$ be a Pythagorean fuzzy value. The degree of indeterminacy is represented by $\pi_{P}(x)$,where:

$$
\pi_{P}(x)=\sqrt{1-\left(\mu_{P}(x)\right)^{2}-\left(v_{P}(x)\right)^{2}} .
$$

Based on the former description, we introduce two score functions to compare the various Pythagorean fuzzy set as follows:

Definition 2.3 (Yager and Abbasov 2013) Let $P=P\left(\mu_{P}, v_{P}\right)$ be a PFS. Then the similarity degree $\mathscr{F}$ for $P$ is defined as follows: 


$$
\mathscr{F}(P)=\frac{1}{2}+\sqrt{\mu_{P}^{2}+v_{P}^{2}} *\left(\frac{1}{2}-\frac{2 \arccos \left(\frac{\mu_{P}}{\sqrt{\mu_{P}^{2}+\mu_{P}^{2}}}\right)}{\pi}\right) .
$$

Definition 2.4 (Sun et al. 2018) Let $P=P\left(\mu_{P}, v_{P}\right)$ be a PFS. Then the similarity degree $\mathscr{A}(P)$ for $P$ is defined as follows:

$$
\mathscr{A}(P)=\mu_{P}-v_{P}
$$

Based on the previous introductions by Definition 2.3 to 2.6, the comparison law for Pythagorean fuzzy sets could be obtained as follows:

Definition 2.5 (Lang et al. 2019) Let $P_{1}=P\left(\mu_{P_{1}}, v_{P_{1}}\right)$ and $P_{2}=P\left(\mu_{P_{2}}, v_{P_{2}}\right)$ be Pythaogrean fuzzy numbers, and $\bullet=\mathscr{F}, \mathscr{A}$. Then

(1) If $\bullet\left(\gamma_{1}\right)>\bullet\left(\gamma_{2}\right)$, then $P_{1}$ is bigger than $P_{2}$, denoted by $\gamma_{1}>$. $\gamma_{2}$;

(2) If $\bullet\left(\gamma_{1}\right)<\bullet\left(\gamma_{2}\right)$, then $P_{1}$ is smaller than $P_{2}$, denoted by $\gamma_{1}<$. $\gamma_{2}$;

(3) If $\bullet\left(\gamma_{1}\right)=\bullet\left(\gamma_{2}\right)$, then $P_{1}$ is equal to $P_{2}$, denoted by $\gamma_{1} \sim . \gamma_{2}$;

\subsection{Rough set and multi-granulation rough set}

In this part, we present the basic concept of rough set model under the framework of two universes. The description of this model could be proposed in (Sun et al. 2016; Pei and Xu 2004).

Definition 2.6 Let $U$ be a infinite universe. $R$ is a relation of equivalence in $U$. For an arbitrary set $X \subseteq U$, the lower approximation and the upper approximation with regards to $X$ are represented by $\underline{R}(X)$ and $R(X)$, which are defined as follows:

$$
\underline{R}(X)=\left\{x \mid[x]_{R} \subseteq X\right\}, \bar{R}(X)=\left\{x \mid[x]_{R} \cap X \neq \varnothing\right\} .
$$

We define $(\underline{R}(X), \bar{R}(X))$ as a rough set concerning a binary relation $R$ in a set $A$.

Additionally, decision makers from various fields may be independent of each other and need multiple binary relation to illustrate the target concept. Qian et al. (2014; 2010a; b) proposed and extended the multi-granulation rough set models.

Definition 2.7 Let $K=(U, A T, f)$ be a complete information system and $P, Q$ be two relations of equivalence on the universe $U$, and $X \subseteq U$. The lower approximation and the upper approximation of $X$ in $U$ are described as follows, specifically:

$$
\underline{X}_{P+Q}=\left\{x \in U \mid[x]_{p} \subseteq X \vee[x]_{Q} \subseteq X\right\}, \bar{X}_{P+Q}=\left(\bar{X}_{P+Q}^{c}\right)^{c} .
$$

Besides, the region of uncertainty of $X$ with reference of the equivalence relation between $P$ and $Q$ is defined as:

$$
B n_{P+Q}=\bar{X}^{P+Q}-\underline{X}_{P+Q}
$$


Briefly, we denote $X$ definable as an equivalence relation between $P$ and $Q$ if $\bar{X}^{P+Q}=\underline{X}_{P+Q}$. Otherwise, $X$ is a rough set concerning $K=(U, A T, f)$.

\section{Grey relational analysis based on Pythagorean fuzzy set and neighborhood rough set}

As the development of human society and economic, the characteristic of data is large volume and information complexity. The main problems for data analysis consist of three aspects, including extracting the key criteria or feature selection, information fusion and result analysis. In this section, we will propose an approach of attribute reduction and a model to obtain the attribute weights.

\subsection{Neighborhood rough set-based attribute reduction}

Attribute reduction plays an important role on data mining for the problem on uncertain circumstance. In recent years, many scholars explored the approaches to study the attribute reduction (Zhang 2005; Wang et al. 2021; Li et al. 2017; Fan et al. 2018). Neighborhood rough set (Hu et al. 2008a) is proposed based on similarity degree. Compared other rough set model, it considers that the objects should be classified in the similar category when the conditional attribute of the objects take similar values, otherwise the classification is not consistent. The other advantage of neighborhood rough set is to improve the tolerance by introducing the parameter $\delta$. Therefore, this paper utilizes the neighborhood rough set to reduct the attributes by $\mathrm{Hu}$ et al. (2008b). The approach improved the original method of neighborhood rough set-based attribute reduction by utilizing the forward search strategy started from the monotonic relationship between the positive region and attribute set and reduces the times of comparison for simples to increase the calculation speed. In the following part, we describe the neighborhood rough set-based attribute reduction.

\subsubsection{Neighborhood rough set}

In the following section, the concept of neighborhood rough set is described and lays the groundwork for the following.

Definition 3.1 Let $\langle U, M, D\rangle$ be a heterogeneous information system, where $U$ is a set of non-empty finite object $U=\left\{x_{1}, x_{2}, \ldots, x_{n},\right\}$, and $M$ is denoted as a value set to describe the characteristic of the object set $U, \mathrm{D}$ as decision attribute. If $M$ could form a neighbor relation on the universe $U$, we call $\langle U, M, D\rangle$ as a neighbor decision system. For given object $x_{i} \in U$ and $B \in M$, then the neighborhood information granule $\delta_{B}\left(x_{i}\right)$ of $x_{i}$ in the condition attribute set $B$ is defined as the distance:

$$
\delta_{B}\left(x_{i}\right)=\left\{x_{j} \in U \mid \Delta^{B}\left(x_{i}, x_{j}\right) \leq \delta\right\},
$$


where $\left\{\delta\left(x_{i}\right) \mid i=1,2, \ldots, n\right\}$ is a cover of $U . \Delta$ is a measurement function.

Definition 3.2 Given the object set $U$ and a neighborhood relation $N$ over the universe $U,\langle U, N\rangle$ can be represented as a neighborhood approximate space. The lower and upper approximate space on an arbitrary $X \in U$ could be defined as follows:

$$
\underline{N X}=\left\{x_{i} \mid \delta\left(x_{i}\right) \subseteq X, x_{i} \in U\right\}, \overline{N X}=\left\{\delta\left(x_{i}\right) \cap X \neq \varnothing, x_{i} \in U\right\} .
$$

\subsubsection{Neighborhood rough set-based attribute reduction}

In this section, we will present the attribute reduction approach based on neighborhood rough set and the algorithm of attribute reduction is described.

Definition 3.3 Let $\langle U, C, D\rangle$ be a neighborhood decision system. $D$ divides $U$ into $N$ equivalence classes: $X_{1}, X_{2}, \ldots, X_{N} . B \subseteq C$ produces a neighbor relation $N_{B}$ on the universe $U$. The lower and upper approximation with regard as $B$ on the decision $D$ is defined as follows:

$$
\underline{N_{B}} D=\left\{\underline{N_{B}} X_{1}, \underline{N_{B}} X_{2}, \ldots, \underline{N_{B}} X_{N}\right\}, \overline{N_{B}} D=\left\{\overline{N_{B}} X_{1}, \overline{N_{B}} X_{2}, \ldots, \overline{N_{B}} X_{N}\right\}
$$

where:

$$
\underline{N_{B}} X=\left\{x_{i} \mid \delta_{B}\left(x_{i}\right) \subseteq X, x_{i} \in U\right\}, \overline{N_{B}} X=\left\{\delta_{B}\left(x_{i}\right) \cap X \neq \varnothing, x_{i} \in U\right\} .
$$

Definition 3.4 Let $\langle U, C, D\rangle$ be a neighborhood decision system. The dependency degree of decision attribute $D$ on conditional attributes $B \subseteq C$ is defined as:

$$
\gamma_{B}(D)=\frac{\operatorname{Card}\left(N_{B} D\right)}{\operatorname{Card}(U)} .
$$

where $\operatorname{Card}(\bullet)$ is denoted as the cardinality of a set.

Definition 3.5 Let $\langle U, C, D\rangle$ be a neighborhood decision system. The importance degree for the attribute $a \in C$ is defined as follows, respectively:

$$
\operatorname{sig}(a, B, D)=\gamma_{B \cup a}(D)-\gamma_{B}(D) .
$$

The reduction algorithm of fast forward attribute selection based on neighborhood rough sets is described as Algorithm 1 proposed by Hu et al. (2008b). 


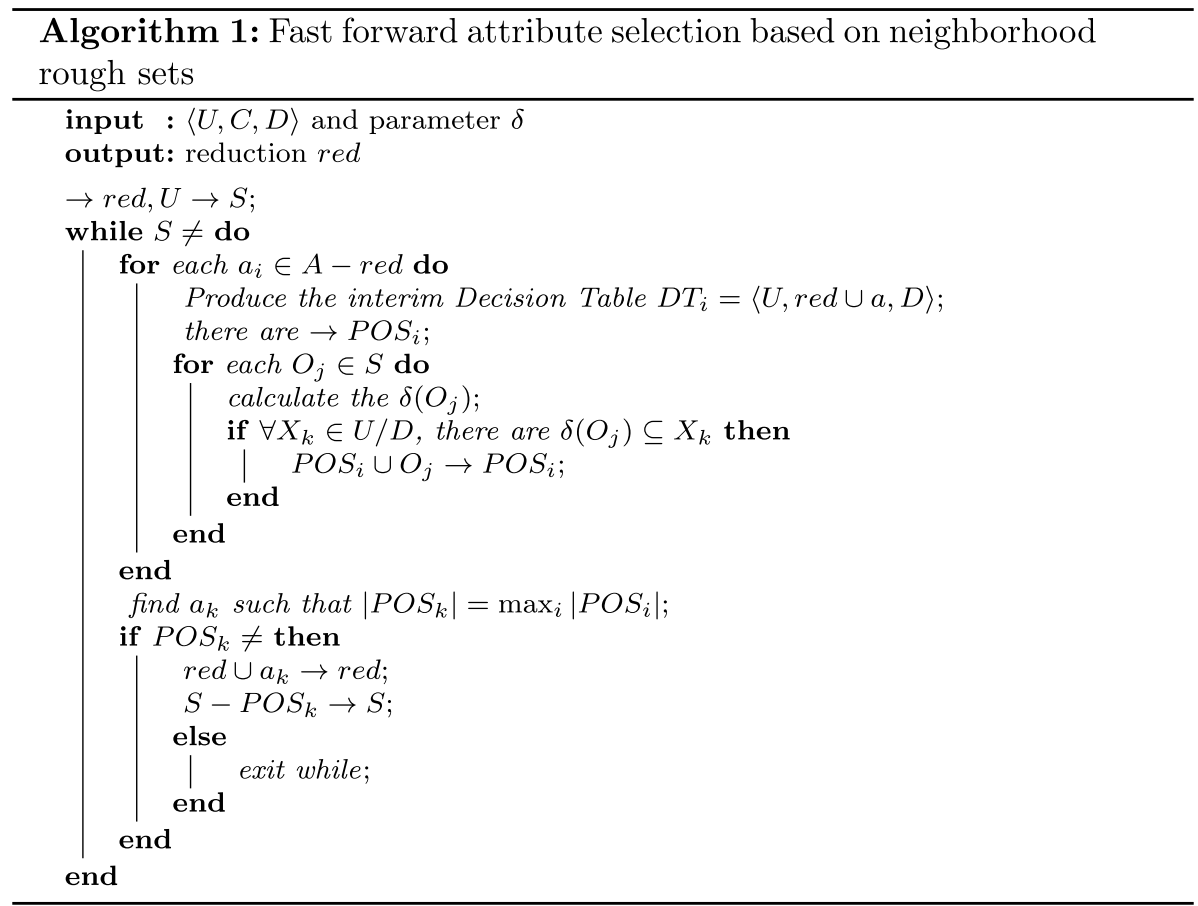

\subsection{Grey relational analysis based on Pythagorean fuzzy set}

Grey relation analysis proposed by Deng (1989) is used to describe the relation between the two variables. Many scholars applied the grey relational analysis to multi-criteria decision making. Khan and Abdullah (2018) proposed a new multi-criteria decision making based on interval-valued Pythagorean fuzzy grey relational analysis and Choquet integral average operator. However, less literatures consider the relational degree from the opposite perspective. The intuitionistic fuzzy set and Pythagorean fuzzy set describe the object by membership degree and non-membership degree. In this paper, we give a new explanation for membership and non-membership degree by combing with relative degree and non-relative degree. In order to describe the fuzzy information on the uncertain circumstance, a new approach to define the membership relation based on the Pythagorean fuzzy set and grey relational analysis is proposed in the following section.

Definition 3.1 Let $X_{0}=\left\{x_{0}(1), x_{0}(2), \ldots, x_{0}(n)\right\}$ and $X_{i}=\left\{x_{i}(1), x_{i}(2), \ldots, x_{i}(n)\right\}$ be two vectors, where $i=1,2, \ldots, m$. The relational degree $\left[\tau\left(x_{0}(k), x_{i}(k)\right), \rho\left(x_{0}(k), x_{i}(k)\right)\right]$ between $X_{0}$ and $X_{i}$ could be defined as follows, respectively: 


$$
\begin{aligned}
& \tau\left(x_{0}(k), x_{i}(k)\right)=\frac{\min _{i} \min _{k}\left|x_{0}(k)-x_{i}(k)\right|+\sigma \max _{i} \max _{k}\left|x_{0}(k)-x_{i}(k)\right|}{\left|x_{0}(k)-x_{i}(k)\right|+\sigma \max _{i} \max _{k}\left|x_{0}(k)-x_{i}(k)\right|}, \\
& \rho\left(x_{0}(k), x_{i}(k)\right)=\frac{\left|x_{0}(k)-x_{i}(k)\right|-\sigma \min _{i} \min _{k}\left|x_{0}(k)-x_{i}(k)\right|}{\left|x_{0}(k)-x_{i}(k)\right|+\sigma \max _{i} \max _{k}\left|x_{0}(k)-x_{i}(k)\right|},
\end{aligned}
$$

where $\sigma \in[0,1]$. The management meaning of $\tau\left(x_{0}(k), x_{i}(k)\right)$ is correlative degree and $\tau\left(x_{0}(k), x_{i}(k)\right)$ is non-correlative degree of two objects.

Property 3.1 The relational degree $\tau\left(x_{0}(k), x_{i}(k)\right)$ and non-relational degree $\rho\left(x_{0}(k), x_{i}(k)\right)$ between $X_{0}$ and $X_{i}$ satisfies the following properties.

(1) $0 \leq \tau\left(x_{0}(k), x_{i}(k)\right) \leq 1,0 \leq \rho\left(x_{0}(k), x_{i}(k)\right) \leq 1$;

(2) $\min _{i} \min _{k}\left|x_{0}(k)-x_{i}(k)\right| \leq\left|x_{0}(k)-x_{i}(k)\right| \leq \max _{i} \max _{k}\left|x_{0}(k)-x_{i}(k)\right|$.

Theorem 3.1 The relational degree $\tau\left(x_{0}(k), x_{i}(k)\right)$ and non-relational degree $\rho\left(x_{0}(k), x_{i}(k)\right)$ between $X_{0}$ and $X_{i}$ satisfies the property of Pythagorean fuzzy set.

$$
0 \leq\left(\tau\left(x_{0}(k), x_{i}(k)\right)\right)^{2}+\left(\rho\left(x_{0}(k), x_{i}(k)\right)\right)^{2} \leq 1 .
$$

By the Definition 3.1, we give a new perspective to describe the Pythagorean fuzzy set considering the correlative degree and non-correlative degree. The correlative degree is denoted as the membership degree and the non-correlative degree is denoted as the nonmembership degree. Then, Theorem 3.1 explores the relation between the correlative degree and non-correlative degree and the proof process is shown as follows, respectively.

Proof Let $a=\min _{i} \min _{k_{k}}\left|x_{0}(k)-x_{i}(k)\right|, b=\max _{i} \max _{k}\left|x_{0}(k)-x_{i}(k)\right|$ and $c=\left|x_{0}(k)-x_{i}(k)\right|$. The equations $\tau \stackrel{i}{=} \frac{a+\delta b}{c+\delta b}$ and $\rho=\frac{c-\delta a}{c+\delta b}$ hold. There are:

$$
\tau^{2}+\rho^{2}=\left(\frac{a+\delta b}{c+\delta b}\right)^{2}+\left(\frac{c-\delta a}{c+\delta b}\right)^{2}=\frac{a^{2}+2 a b \delta+\delta^{2} b+c^{2}-2 a c \delta+\delta^{2} a^{2}}{(c+\delta b)^{2}}=\frac{B}{A},
$$

Let $\Gamma=B-A=a^{2}-2 a b \delta-2 a c \delta+\delta^{2} a^{2}-2 b c \delta=\left(1+\delta^{2}\right) a^{2}+2 a \delta(b-c)-2 b c \delta$, $\Delta=(2 \delta(b-c))^{2}+8 b c \delta\left(1+\delta^{2}\right) \geq 0$, when $\Gamma=0$, there are :

$$
\begin{aligned}
& a_{1}=\frac{-2 \delta(b-c)-\sqrt{8 b c \delta\left(1+\delta^{2}\right)+[2 \delta(b-c)]^{2}}}{2\left(1+\delta^{2}\right)}<0, \text { and } \\
& a_{2}=\frac{-2 \delta(b-c)+\sqrt{8 b c \delta\left(1+\delta^{2}\right)+[2 \delta(b-c)]^{2}}}{2\left(1+\delta^{2}\right)},
\end{aligned}
$$

$2 \delta(b-c) \leq \sqrt{8 b c \delta\left(1+\delta^{2}\right)+[2 \delta(b-c)]^{2}} \leq \sqrt{8 b c \delta\left(1+\delta^{2}\right)}+2 \delta(b-c), 0<a_{2} \leq 1$,

When $a_{1}<0 \leq a \leq a_{2}$, the value of $\Gamma \leq 0$, the equation $\tau^{2}+\rho^{2} \leq 1$.

Therefore, The pair set $\left(\tau\left(x_{0}(k), x_{i}(k)\right), \rho\left(x_{0}(k), x_{i}(k)\right)\right)$ is satisfied with the condition of Pythagorean fuzzy set. where $\tau\left(x_{0}(k), x_{i}(k)\right)$ is denoted as membership degree and $\rho\left(x_{0}(k), x_{i}(k)\right)$ is denoted as non-membership degree. 


\section{Multi-granulation Pythagorean fuzzy rough set over two universes}

As the uncertainty and complexity for our life increase, the crisp number is no longer satisfied with the real world. Furthermore, individuals are usually bounded rationality and have different attitudes facing the risks, including optimistic, pessimistic and neutral. Accordingly, we will propose multi-granulation Pythagorean fuzzy rough set combined with the risk attitudes of various individuals under the framework of multiple granular fuzzy approximate space on two universes considering the risk attitudes.

In this section, rough approximation concept of the Pythagorean fuzzy set will be discussed in regards as multiple granularity fuzzy approximate space. We will present three various multi-granulation Pythagorean fuzzy rough set models under the framework of two universes according to the corresponding decision making model.

The rough approximate concept of a Pythagorean fuzzy set on the multiple granularity fuzzy approximate space under the framework of two universes is determined by a family of binary compatibility mapping between $U$ and $V$. Nevertheless, there may be a family of binary fuzzy relations under the framework of two universes as a result of the increasing complexity of the socioeconomic environment. Furthermore, the multiple granularity fuzzy approximate space under the framework of two universes is proposed. This subsection will focus on the multiple granularity rough approximate concept of a Pythagorean fuzzy set under the framework of two universes, and optimistic multi-granulation Pythagorean fuzzy rough set, pessimistic multi-granulation Pythagorean fuzzy rough set and variable precision multi-granulation Pythagorean fuzzy rough set on the multiple granularity fuzzy approximate space under the framework of two universes.

First, we explore the optimistic multi-granulation Pythagorean fuzzy rough set on the multiple granularity fuzzy approximate space under the framework of two universes.

\subsection{Optimistic multi-granulation Pythagorean fuzzy rough set over two universes}

For the optimists, they usually consider the positive side and believe that the candidates are optimal as long as an individual is satisfied finds it appropriate or an attribute for an individual is in line with forecast. From the perspective, this section presents an optimistic multi-granulation Pythagorean fuzzy rough set over two universes. Firstly, we give the definition of the optimistic multi-granulation Pythagorean fuzzy rough set.

Definition 4.1 Let $(U, V, \mathbf{R})$ be the multiple granularity fuzzy approximate space under the framework of two universes. For a Pythagorean fuzzy set $P$ of the universe $V$, $P=\left[\tau_{P}(y), \rho_{P}(y)\right], y \in V, x \in U$. The optimistic lower approximate set $\underline{\mathbf{R}}_{\sum_{i=1}^{m} R_{i}}^{O}(P)$ and upper approximate set $\overline{\mathbf{R}}_{\sum_{i=1}^{m} R_{i}}^{O}(P)$ of a Pythagorean fuzzy set on the space $(U, V, \mathbf{R})$ are defined as follows, respectively:

$$
\begin{aligned}
& \underline{\mathbf{R}}_{\sum_{k=1}^{m} R_{i}}^{O}(P)(x)=\left[\mu_{\underline{\mathbf{R}}_{\Sigma_{i=1}^{m} R_{i}}^{O}(P)}, v_{\mathbf{R}_{\Sigma_{i=1}^{m} R_{i}}^{O}(P)}\right], x \in U, \\
& \overline{\mathbf{R}}_{\sum_{i=1}^{m} R_{i}}^{O}(P)(x)=\left[\mu_{\overline{\mathbf{R}}_{\sum_{i=1}^{O} R_{i}}^{O}(P)}, v_{\overline{\mathbf{R}}_{\sum_{i=1}^{O} R_{i}}^{O}(P)}\right], x \in U,
\end{aligned}
$$

where: 


$$
\begin{aligned}
\mu_{\underline{\mathbf{R}}_{\Sigma_{i=1}^{m} R_{i}}^{o}(P)} & =\bigvee_{i=1}^{m} \bigwedge_{y \in V}\left[1-R_{i}(x, y) \vee \tau_{P}(y)\right], \\
v_{\underline{\mathbf{R}}_{\Sigma_{i=1}^{m} R_{i}}^{o}(P)} & =\bigwedge_{i=1}^{m} \bigvee_{y \in V}\left[R_{i}(x, y) \vee \rho_{P}(y)\right], \\
\mu_{\overline{\mathbf{R}}_{\Sigma_{i=1}^{m} R_{i}}^{O}(P)} & =\bigwedge_{i=1}^{m} \bigvee_{y \in V}\left[R_{i}(x, y) \vee \tau_{P}(y)\right], \\
v_{\overline{\mathbf{R}}_{\Sigma_{i=1}^{o} R_{i}}^{O}(P)} & =\bigvee_{i=1}^{m} \bigwedge_{y \in V}\left[1-R_{i}(x, y) \vee \rho_{P}(y)\right] .
\end{aligned}
$$

Particularly, when universes $U$ and $V$ are infinite sets, the operators $\bigvee$ and $\bigwedge$ will become inf and sup.

Theorem 4.1 Let $P$ be a Pythagorean fuzzy set of $U$ and $R$ be a binary fuzzy relation on $U \times V . \underline{\mathbf{R}}$ and $\overline{\mathbf{R}}$ are the lower and upper approximation of $P$ in regards as $(U, V, \mathbf{R})$. For any $x \in U$, the membership degree and non-membership degree of the approximation operators satisfy the following relationship.

(1) $0 \leq\left(\mu_{\underline{\mathbf{R}}_{\Sigma_{i=1}^{m} R_{i}}^{o}(P)}\right)^{2}+\left(v_{\mathbf{R}_{\Sigma_{i=1}^{m} R_{i}}^{o}(P)}\right)^{2} \leq 1$

(2) $0 \leq\left(\mu_{\overline{\mathbf{R}}_{\sum_{i=1}^{m} R_{i}}^{o}(P)}\right)^{2}+\left(v_{\overline{\mathbf{R}}_{\sum_{i=1}^{m} R_{i}}^{o}(P)}\right)^{2} \leq 1$.

Proof (1) By definition 4.1, for any $x \in U, y \in V$, we have:

$$
\begin{aligned}
1-\left(v_{\underline{\mathbf{R}}_{\Sigma_{i=1}^{m} R_{i}}^{o}(P)}\right)^{2} & =1-\bigwedge_{i=1}^{m} \bigvee_{y \in V}\left[R_{i}(x, y) \vee\left(\rho_{P}(y)\right)^{2}\right] \\
& =\bigvee_{i=1}^{m}\left[\bigwedge_{y \in V}\left(1-R_{i}(x, y)\right) \vee\left(1-\rho_{P}^{2}(y)\right)\right] \\
& \left.\geq \bigvee_{i=1}^{m} \bigwedge_{y \in V}\left[1-R_{i}(x, y)\right) \vee \gamma_{P}^{2}(y)\right]=\left(\mu_{\underline{\mathbf{R}}_{\Sigma_{i=1}^{o} R_{i}}^{o}(P)}\right)^{2},
\end{aligned}
$$

This is the process of proof.

(2) is similar to (1).

It is easy to know that $\underline{\mathbf{R}}_{\sum_{i=1}^{m} R_{i}}^{O}(P)$ and $\overline{\mathbf{R}}_{\sum_{i=1}^{m} R_{i}}^{O}(P)$ are two Pythagorean fuzzy sets on the universe $U$. Furthermore, $P$ is called as a definable Pythagorean fuzzy set on the multiple granularity fuzzy approximate space under the framework of two universes $(U, V, \mathbf{R})$ when $\underline{\mathbf{R}}_{\sum_{i=1}^{m} R_{i}}^{O}(P)=\overline{\mathbf{R}}_{\sum_{i=1}^{m} R_{i}}^{O}(P)$,i.e., $\mu_{\underline{\mathbf{R}}_{\sum_{i=1}^{m} R_{i}}^{O}(P)}=\mu_{\overline{\mathbf{R}}_{\sum_{i=1}^{o} R_{i}}^{o}(P)}$ and $v_{\underline{\mathbf{R}}_{\sum_{i=1}^{m} R_{i}}^{o}(P)}=v_{\overline{\mathbf{R}}_{\sum_{i=1}^{m} R_{i}}^{o}(P)}$ for any $x \in U$. Otherwise, we denote the set-pair $\left(\underline{\mathbf{R}}_{\sum_{i=1}^{m} R_{i}}^{O}(P), \overline{\mathbf{R}}_{\sum_{i=1}^{m} R_{i}}^{O}(P)\right)$ as the optimistic multigranulation Pythagorean fuzzy rough set under the framework of two universes.

Remark 4.1 Let $(U, V, \mathbf{R})$ be the multiple granularity fuzzy approximate space under the framework of two universes. For a Pythagorean fuzzy set $P$ of the universe $V$, 
$P=\left[\tau_{P}(y), \rho_{P}(y)\right], y \in V, x \in U$. The relation $\underline{\mathbf{R}}_{\sum_{k=1}^{m} R_{i}}^{O}(P)(x) \subseteq \overline{\mathbf{R}}_{\sum_{k=1}^{m} R_{i}}^{O}(P)(x)$ are not satisfied based on the above definitions.

Above all, the relation $\underline{\mathbf{R}}_{\sum_{k=1}^{m} R_{i}}^{O}(P)(x) \subseteq \overline{\mathbf{R}}_{\sum_{k=1}^{m} R_{i}}^{O}(P)(x)$ will be satisfied when $U=V$. Additionally, we could get the following these conclusion.

Proposition 4.1 Let $(U, V, \mathbf{R})$ be the multiple granularity fuzzy approximate space under the framework of two universes. For a Pythagorean fuzzy set $P$ of the universe $V$, $P=\left[\tau_{P}(y), \rho_{P}(y)\right], y \in V, x \in U$. If $R_{i} \in \mathbf{R}(i=1,2, \ldots, m)$ are fuzzy binary relationships from $U$ to $V$, then $\underline{\mathbf{R}}_{\sum_{k=1}^{m} R_{i}}^{O}(P)(x) \subseteq \overline{\mathbf{R}}_{\sum_{k=1}^{m} R_{i}}^{O^{\prime}}(P)(x)$.

Remark 4.2 Let $(U, V, \mathbf{R})$ be the multiple granularity fuzzy approximate space under the framework of two universes. For a Pythagorean fuzzy set $P$ of the universe $V$, $P=\left[\tau_{P}(y), \rho_{P}(y)\right], y \in V, x \in U$. Some conclusions could be obtained:

(1) If $R_{i} \in \mathbf{R}$ is a binary compatibility relationship derived from binary mapping $F_{i}$ from universe $U$ to $V$, then $R_{i}(x, y) \Leftrightarrow F_{i}(x)=\left\{y \in V \mid(x, y) \in R_{i}\right\}$. Therefore, $\underline{\mathbf{R}}_{\sum_{k=1}^{m} R_{i}}^{O}(P)(x)$ and the degeneration from $\overline{\mathbf{R}}_{\sum_{k=1}^{m} R_{i}}^{O}(P)(x)$ into $\underline{R}_{\sum_{i=1}^{m} R_{i}}^{O}(P)$ and $\overline{\mathfrak{R}}_{\sum_{i=1}^{m} R_{i}}^{O}(P)$ could be obtained.

(2) If $R_{1}=R_{2}=\cdots=R_{m}$, for any $x \in U$ and $y \in V$,

$$
\begin{aligned}
& \mu_{\underline{\mathbf{R}}_{R_{i}}^{o}(P)}=\bigwedge_{y \in V}\left[1-R_{i}(x, y) \vee \tau_{P}(y)\right], \quad v_{\mathbf{R}_{R_{i}}^{o}(P)}=\bigvee_{y \in V}\left[R_{i}(x, y) \vee \rho_{P}(y)\right], \\
& \mu_{\overline{\mathbf{R}}_{R_{i}}^{o}(P)}=\bigvee_{y \in V}\left[R_{i}(x, y) \vee \tau_{P}(y)\right], \quad v_{\overline{\mathbf{R}}_{R_{i}}^{o}(P)}=\bigwedge_{y \in V}\left[1-R_{i}(x, y) \vee \rho_{P}(y)\right] .
\end{aligned}
$$

(3) Supposed that $P$ is a fuzzy set on the universe $V$, then optimistic multi-granulation Pythagorean fuzzy rough set degenerates into multi-granulation fuzzy rough set under the framework of two universes. Besides, the optimistic multi-granulation Pythagorean fuzzy rough set will degenerate into the fuzzy rough set under the framework of two universes by $\mathrm{Wu}$ et al. (2003) when $R_{1}=R_{2}=\cdots=R_{m}$ and the classical fuzzy rough set when $U=V$.

Proposition 4.2 Let $(U, V, \mathbf{R})$ be the multiple granularity fuzzy approximate space under the framework of two universes. For a Pythagorean fuzzy set $P$ of the universe $V$, $P=\left[\tau_{P}(y), \rho_{P}(y)\right], y \in V$. Then, some results could be attained:

(1) $\underline{\mathbf{R}}_{\sum_{k=1}^{m} R_{i}}^{O}(P)=\bigcup_{i=1}^{m} \underline{\underline{\mathbf{R}_{i}}}(A)$;

(2) $\overline{\mathbf{R}}_{\sum_{k=1}^{m} R_{i}}^{\sigma^{k=1}}(P)=\bigcap_{i=1}^{m} \overline{\mathbf{R}_{i}}(A)$.

Theorem 4.2 Let $(U, V, \mathbf{R})$ be the multiple granularity fuzzy approximate space under the framework of two universes. For a Pythagorean fuzzy set $P$ of universe $V$, $P=\left[\tau_{P}(y), \rho_{P}(y)\right], y \in V$. Then, some results could be attained:

(1) $\underline{\mathbf{R}}_{\sum_{i=1}^{m} R_{i}}^{O}\left(P^{C}\right)=\left(\overline{\mathbf{R}}_{\sum_{i=1}^{m} R_{i}}^{O}(P)\right)^{C}, \quad \overline{\mathbf{R}}_{\sum_{i=1}^{m} R_{i}}^{O}\left(P^{C}\right)=\left(\underline{\mathbf{R}}_{\sum_{i=1}^{m} R_{i}}^{O}(P)\right)^{C}$; 
(2) $\underline{\mathbf{R}}_{\sum_{i=1}^{m} R_{i}}^{O}\left(P_{1} \cup P_{2}\right) \supseteq \underline{\mathbf{R}}_{\sum_{i=1}^{m} R_{i}}^{O}\left(P_{1}\right) \cup \underline{\mathbf{R}}_{\sum_{i=1}^{m} R_{i}}^{O}\left(P_{2}\right), \overline{\mathbf{R}}_{\sum_{i=1}^{m} R_{i}}^{O}\left(P_{1} \cap P_{2}\right) \subseteq \overline{\mathbf{R}}_{\sum_{i=1}^{m} R_{i}}^{O}\left(P_{1}\right) \cap \overline{\mathbf{R}}_{\sum_{i=1}^{m} R_{i}}^{O}\left(P_{2}\right)$;

(3) $\overline{\overline{\mathbf{R}}} \sum_{i=1}^{m} R_{i}\left(P_{1} \cup P_{2}\right)=\overline{\mathbf{R}}_{\sum_{i=1}^{m} R_{i}}^{\sigma_{i=1}}\left(P_{1}\right) \cup \overline{\mathbf{R}}_{\sum_{i=1}^{m} R_{i}}^{\sigma_{i=1} R_{i}}\left(P_{2}\right), \underline{\mathbf{R}}_{\sum_{i=0}^{m} R_{i}}^{O}\left(P_{1} \cap P_{2}\right)=\underline{\mathbf{R}}_{\sum_{i=1}^{m} R_{i}}^{O}\left(P_{1}\right) \cap \underline{\mathbf{R}}_{\sum_{i=1}^{m} R_{i}}^{O}\left(P_{2}\right)$;

(4) If $P_{1} \subseteq P_{2}$, then $\underline{\mathbf{R}}_{\sum_{i=1}^{m} R_{i}}^{O}\left(P_{1}\right) \subseteq \underline{\mathbf{R}}_{\sum_{i=1}^{m} R_{i}}^{O}\left(P_{2}\right), \overline{\mathbf{R}}_{\sum_{i=1}^{m} R_{i}}\left(P_{1}\right) \subseteq \overline{\mathbf{R}}_{\sum_{i=1}^{m} R_{i}}^{O}\left(P_{2}\right)$.

\subsection{Pessimistic multi-granulation Pythagorean fuzzy rough set over two universes}

For the pessimists, they hope that the optimal alternatives are satisfied with all the decision makers or an attribute is in line with the expectation of individuals. In this subsection, we will present the pessimistic multi-granulation Pythagorean fuzzy rough set on the multiple granularity fuzzy approximate space under the framework of two universes.

Definition 4.2 Let $(U, V, \mathbf{R})$ be the multiple granularity fuzzy approximate space under the framework of two universes. For a Pythagorean fuzzy set $P$ of universe $V$, $P=\left[\tau_{P}(y), \rho_{P}(y)\right], y \in V, x \in U$. The pessimistic lower approximate set $\underline{\mathbf{R}}_{\sum_{i=1}^{m} R_{i}}^{P}(P)$ and upper approximate set $\overline{\mathbf{R}}_{\sum_{i=1}^{m} R_{i}}^{P}(P)$ of a Pythagorean fuzzy set on the approximate space $(U, V, \mathbf{R})$ are defined as follows, respectively:

$$
\begin{aligned}
& \underline{\mathbf{R}}_{\sum_{k=1}^{m} R_{i}}^{P}(P)(x)=\left[\mu_{\underline{\mathbf{R}}_{\sum_{i=1}^{m} R_{i}}^{P}(P)}, v_{\mathbf{R}_{\sum_{i=1}^{m} R_{i}}^{P}(P)}\right], x \in U, \\
& \overline{\mathbf{R}}_{\sum_{i=1}^{m} R_{i}}^{P}(P)(x)=\left[\mu_{\overline{\mathbf{R}}_{\sum_{i=1}^{P} R_{i}}(P)}, v_{\overline{\mathbf{R}}_{\sum_{i=1}^{P} R_{i}}^{P}(P)}\right], x \in U,
\end{aligned}
$$

where

$$
\begin{aligned}
\mu_{\underline{\mathbf{R}}_{\sum_{i=1}^{m} R_{i}}^{P}}(P) & =\bigwedge_{i=1}^{m} \bigwedge_{y \in V}\left[1-R_{i}(x, y) \vee \tau_{P}(y)\right], \\
v_{\underline{\mathbf{R}}_{\sum_{i=1}^{m} R_{i}}^{P}(P)} & =\bigvee_{i=1}^{m} \bigvee_{y \in V}\left[R_{i}(x, y) \vee \rho_{P}(y)\right], \\
\mu_{\overline{\mathbf{R}}_{\Sigma_{i=1}^{m} R_{i}}(P)} & =\bigvee_{i=1}^{m} \bigvee_{y \in V}\left[R_{i}(x, y) \vee \tau_{P}(y)\right], \\
v_{\overline{\mathbf{R}}_{\Sigma_{i=1}^{m} R_{i}}(P)} & =\bigwedge_{i=1}^{m} \bigwedge_{y \in V}\left[1-R_{i}(x, y) \vee \rho_{P}(y)\right] .
\end{aligned}
$$

Proposition 4.3 Let $(U, V, \mathbf{R})$ be the multiple granularity fuzzy approximate space under the framework of two universes. For a Pythagorean fuzzy set $P$ of the universe $V$, $P=\left[\tau_{P}(y), \rho_{P}(y)\right], y \in V$. Then, some interesting conclusions could be obtained:

(1) $\underline{\mathbf{R}}_{P^{m}{ }^{m} R_{i}}^{P}(P)=\bigcap_{i=1}^{m} \underline{\underline{R_{i}}}(A)$;

(2) $\overline{\mathbf{R}}_{\sum_{k=1}^{m} R_{i}}(P)=\bigcup_{i=1}^{m} \overline{R_{i}}(A)$. 
Theorem 4.3 Let a triple set $(U, V, \mathbf{R})$ be the multiple granularity approximate space under the framework of two universes for any Pythagorean fuzzy sets $P_{1}$ and $P_{2}$ in the universe $V$. Then, some interesting results could be obtained:

(1) $\underline{\mathbf{R}}_{\sum_{i=1}^{m} R_{i}}^{P}(P) \subseteq U, \subseteq \overline{\mathbf{R}}_{\sum_{i=1}^{m} R_{i}}^{P}(P) \subseteq U$,

(2) $\quad \underline{\mathbf{R}}_{\sum_{i=1}^{m} R_{i}}^{P}\left(P^{C}\right)=\left(\overline{\mathbf{R}}_{\sum_{i=1}^{m} R_{i}}^{P}(P)\right)^{C}, \overline{\mathbf{R}}_{\sum_{i=1}^{m} R_{i}}^{P}\left(P^{C}\right)=\left(\underline{\mathbf{R}}_{\sum_{i=1}^{m} R_{i}}^{P}(P)\right)^{C}$;

(3) $\underline{\mathbf{R}}_{\sum_{i=1}^{m} R_{i}}^{P_{i=1}}\left(P_{1} \cup P_{2}\right) \supseteq \underline{\mathbf{R}}_{\sum_{i=1}^{m} R_{i}}^{P}\left(P_{1}\right) \cup \underline{\mathbf{R}}_{\sum_{i=1}^{m} R_{i}}^{P_{i=1}}\left(P_{2}\right), \overline{\mathbf{R}}_{\sum_{i=1}^{m} R_{i}}^{P}\left(P_{1} \cap P_{2}\right) \subseteq \overline{\mathbf{R}}_{\sum_{i=1}^{m} R_{i}}^{P}\left(P_{1}\right) \cap \overline{\mathbf{R}}_{\sum_{i=1}^{m} R_{i}}^{P}\left(P_{2}\right)$;

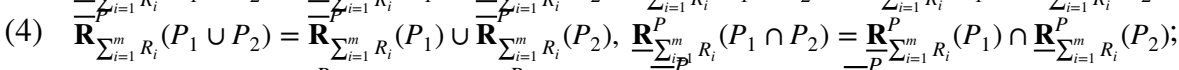

(5) If $P_{1} \subseteq P_{2}$, then $\underline{\mathbf{R}}_{\sum_{i=1}^{m} R_{i}}^{P}\left(P_{1}\right) \subseteq \underline{\mathbf{R}}_{\sum_{i=1}^{P} R_{i}}^{P}\left(P_{2}\right), \overline{\mathbf{R}}_{\sum_{i=1}^{m} R_{i}}\left(P_{1}\right) \subseteq \overline{\mathbf{R}}_{\sum_{i=1}^{m} R_{i}}^{P}\left(P_{2}\right)$.

\subsection{Variable precision multi-granulation Pythagorean fuzzy rough set over two universes}

For the optimistic and pessimistic multi-granulation Pythagorean fuzzy rough set over two universes, two perspectives only consider two extreme situations. However, the absolute optimism and pessimism are hardly satisfied with the actual situation and the optimal candidates requires only a majority of individuals to agree. To ease conditions, we propose two variable precision multi-granulation Pythagorean fuzzy rough sets according to the optimistic and pessimistic multi-granulation Pythagorean fuzzy rough set, in regards as the multiple granularity fuzzy approximate space under the framework of two universes.

Definition 4.3 (Type - I Variable precision multi-granulation Pythagorean fuzzy rough set) Let $(U, V, \mathbf{R})$ be the multiple granularity fuzzy approximate space under the framework of two universes. For a Pythagorean fuzzy set $P$ of universe $V, P=\left[\tau_{P}(y), \rho_{P}(y)\right], y \in V$ and $\alpha \in[0,1]$. The variable precision lower approximate set $(I) \underline{\mathbf{R}}_{\sum_{i=1}^{m} R_{i}}^{\alpha}(P)$ and upper approximate set $(I) \overline{\mathbf{R}}_{\sum_{i=1}^{m} R_{i}}^{\alpha}(P)$ of a Pythagorean fuzzy set on the space $(U, V, \mathbf{R})$ are defined as follows, respectively:

$$
\begin{aligned}
& (I) \underline{\mathbf{R}}_{\sum_{k=1}^{m} R_{i}}^{\alpha}(P)(x)=\left[\mu_{\underline{\mathbf{R}}_{\Sigma_{i=1}^{P} R_{i}}^{P}(P)}, v_{\mathbf{\mathbf { R }}_{\sum_{i=1}^{m} R_{i}}^{P}(P)}\right], x \in U, \\
& (I) \overline{\mathbf{R}}_{\sum_{i=1}^{m} R_{i}}(P)(x)=\left[\mu_{\overline{\mathbf{R}}_{\Sigma_{i=1}^{m} R_{i}}^{\alpha}(P)}, v_{\overline{\mathbf{R}}_{\sum_{i=1}^{m} R_{i}}^{\alpha}(P)}\right], x \in U,
\end{aligned}
$$

where $x \in U, y \in V$ and: 


$$
\begin{aligned}
\mu_{\underline{\mathbf{R}}_{\Sigma_{i=1}^{m} R_{i}}^{\alpha}}(P) & =\bigvee_{i=1}^{m}\left[\bigwedge_{\tau_{P}(y) \leq \alpha}\left(1-R_{i}(x, y) \vee \alpha\right) \wedge \bigwedge_{\tau_{P}(y)>\alpha}\left(\left(1-R_{i}(x, y)\right) \vee \tau_{P}(y)\right)\right], \\
v_{\underline{\mathbf{R}}_{\sum_{i=1}^{m} R_{i}}^{\alpha}(P)} & =\bigwedge_{i=1}^{m}\left[\bigvee_{\rho_{P}(y) \geq(1-\alpha)}\left(R_{i}(x, y) \wedge(1-\alpha)\right) \vee \bigvee_{\rho_{P}(y)<(1-\alpha)}\left(R_{i}(x, y) \wedge \rho_{P}(y)\right)\right], \\
\mu_{\overline{\mathbf{R}}_{\Sigma_{i=1}^{\alpha} R_{i}}^{\alpha}(P)} & =\bigwedge_{i=1}^{m}\left[\bigvee_{\tau_{P}(y) \geq(1-\alpha)}\left(R_{i}(x, y) \wedge(1-\alpha)\right) \vee \bigvee_{\tau_{P}(y)<(1-\alpha)}\left(R_{i}(x, y) \wedge \tau_{P}(y)\right)\right], \\
v_{\overline{\mathbf{R}}_{\Sigma_{i=1}^{\alpha} R_{i}}(P)} & =\bigvee_{i=1}^{m}\left[\bigwedge_{\rho_{P}(y) \leq \alpha}\left(1-R_{i}(x, y) \vee \alpha\right) \wedge \bigwedge_{\rho_{P}(y)>\alpha}\left(\left(1-R_{i}(x, y)\right) \vee \rho_{P}(y)\right)\right],
\end{aligned}
$$

Respectively, when universes $U$ and $V$ are infinite sets, the operators $\bigvee$ and $\bigwedge$ will become inf and sup.

It is easy to obtain that $(I) \underline{\mathbf{R}}_{\sum_{i=1}^{m} R_{i}}^{\alpha}(P)$ and $(I) \overline{\mathbf{R}}_{\sum_{i=1}^{m} R_{i}}^{\alpha}(P)$ are two PFSs on the universes $U$. Additionally, $P$ is called a definable PFS on the multiple granularity fuzzy approximate space under the framework of two universes $(U, V, \mathbf{R})$ with parameter $\alpha$ when $(I) \underline{\mathbf{R}}_{\sum_{i=1}^{m} R_{i}}^{\alpha}(P)=(I) \overline{\mathbf{R}}_{\sum_{i=1}^{\alpha} R_{i}}^{\alpha}(P)$. i.e., $\mu_{\underline{\mathbf{R}}_{\sum_{i=1}^{m} R_{i}}^{\alpha}(P)}=\mu_{\overline{\mathbf{R}}_{\sum_{i=1}^{\alpha} R_{i}}^{\alpha}(P)}$ and $v_{\underline{\mathbf{R}}_{\Sigma_{i=1}^{\alpha} R_{i}}^{\alpha}(P)}=v_{\overline{\mathbf{R}}_{\sum_{i=1}^{\alpha} R_{i}}^{\alpha}(P)}$ for any $x \in U$. If not, we call the set pair $\left.(I) \underline{\mathbf{R}}_{\sum_{i=1}^{m} R_{i}}^{\alpha}(P),(I) \overline{\mathbf{R}}_{\sum_{i=1}^{m} R_{i}}^{\alpha}(P)\right)$ the $I$ variable precision multi-granulation Pythagorean fuzzy rough set under the framework of two universes.

Definition 4.4 (Type - II Variable precision multi-granulation Pythagorean fuzzy rough set) Let $(U, V, \mathbf{R})$ be the multiple granularity fuzzy approximate space under the framework of two universes. For a Pythagorean fuzzy set $P$ of universe $V, P=\left[\tau_{P}(y), \rho_{P}(y)\right], y \in V$ and $\alpha \in[0,1]$. The variable precision lower approximate set $(I I) \underline{\mathbf{R}}_{\sum_{i=1}^{\alpha} R_{i}}^{\alpha}(P)$ and upper approximate set $(I I) \overline{\mathbf{R}}_{\sum_{i=1}^{m} R_{i}}^{\alpha}(P)$ of a Pythagorean fuzzy set in $(U, V, \mathbf{R})$ are defined as follows, respectively:

$$
\begin{aligned}
& (I I) \underline{\mathbf{R}}_{\sum_{k=1}^{m} R_{i}}^{\alpha}(P)(x)=\left[\mu_{\mathbf{R}_{\sum_{i=1}^{m} R_{i}}^{P}(P)}, v_{\mathbf{R}_{\sum_{i=1}^{P} R_{i}}^{P}(P)}\right], x \in U, \\
& (I I) \overline{\mathbf{R}}_{\sum_{i=1}^{m} R_{i}}(P)(x)=\left[\mu_{\overline{\mathbf{R}}_{\Sigma_{i=1}^{\alpha} R_{i}}^{\alpha}(P)}, v_{\overline{\mathbf{R}}_{\sum_{i=1}^{m} R_{i}}^{\alpha}(P)}\right], x \in U,
\end{aligned}
$$

where $x \in U, y \in V$ and:

$$
\begin{aligned}
& \mu_{\underline{\mathbf{R}}_{\Sigma_{i=1}^{m} R_{i}}^{\alpha}(P)}=\bigwedge_{i=1}^{m}\left[\bigwedge_{\tau_{P}(y) \leq \alpha}\left(1-R_{i}(x, y) \vee \alpha\right) \wedge \bigwedge_{\tau_{P}(y)>\alpha}\left(\left(1-R_{i}(x, y)\right) \vee \tau_{P}(y)\right)\right], \\
& v_{\underline{\mathbf{R}}_{i=1}^{\alpha} R_{i}}(P) \\
& =\bigvee_{i=1}^{m}\left[\bigvee_{\rho_{P}(y) \geq(1-\alpha)}\left(R_{i}(x, y) \wedge(1-\alpha)\right) \vee \bigvee_{\rho_{P}(y)<(1-\alpha)}\left(R_{i}(x, y) \wedge \rho_{P}(y)\right)\right], \\
& \mu_{\overline{\mathbf{R}}_{\Sigma_{i=1}^{m} R_{i}}^{\alpha}(P)}=\bigvee_{i=1}^{m}\left[\bigvee_{\tau_{P}(y) \geq(1-\alpha)}\left(R_{i}(x, y) \wedge(1-\alpha)\right) \vee \bigvee_{\tau_{P}(y)<(1-\alpha)}\left(R_{i}(x, y) \wedge \tau_{P}(y)\right)\right], \\
& v_{\overline{\mathbf{R}}_{\Sigma_{i=1}^{\alpha} R_{i}}(P)}=\bigwedge_{i=1}^{m}\left[\bigwedge_{\rho_{P}(y) \leq \alpha}\left(1-R_{i}(x, y) \vee \alpha\right) \wedge \bigwedge_{\rho_{P}(y)>\alpha}\left(\left(1-R_{i}(x, y)\right) \vee \rho_{P}(y)\right)\right] .
\end{aligned}
$$


Respectively, when universes $U$ and $V$ are infinite sets, the operators $\bigvee$ and $\bigwedge$ will become inf and sup.

It is easy to obtain that $(I I) \underline{\mathbf{R}}_{\sum_{i=1}^{m} R_{i}}^{\alpha}(P)$ and $(I I) \overline{\mathbf{R}}_{\sum_{i=1}^{m} R_{i}}^{\alpha}(P)$ are two Pythagorean fuzzy rough sets on the universe $U$. Additionally, $P$ is called as a definable Pythagorean fuzzy set on multiple granularity fuzzy approximate space under the framework of two universes $(U, V, \mathbf{R})$ with parameter $\alpha$ when $(I I) \underline{\mathbf{R}}_{\sum_{i=1}^{m} R_{i}}^{\alpha}(P)=(I I) \overline{\mathbf{R}}_{\sum_{i=1}^{\alpha} R_{i}}^{\alpha}(P)$. i.e., $\mu_{\underline{\mathbf{R}}_{\sum_{i=1}^{m} R_{i}}^{\alpha}(P)}=\mu_{\overline{\mathbf{R}}_{\sum_{i=1}^{\alpha} R_{i}}^{\alpha}(P)}$ and $\quad v_{\mathbf{R}_{\Sigma_{i=1}^{m} R_{i}}^{\alpha}(P)}=v_{\overline{\mathbf{R}}_{\sum_{i=1}^{\alpha} R_{i}}^{\alpha}(P)}$ for any $x \in U$. If not, we call the set pair $\left.(I I) \underline{\mathbf{R}}_{\sum_{i=1}^{m} R_{i}}^{\alpha}(P),(I I) \overline{\mathbf{R}}_{\sum_{i=1}^{m} R_{i}}^{\alpha_{i=1} R_{i}}(P)\right)$ the Type - II variable precision multi-granulation Pythagorean fuzzy rough set under the framework of two universes.

Similar to optimistic and pessimistic multi-granulation Pythagorean fuzzy rough set under the framework of two universes, we could obtain that both relations $(I) \underline{\mathbf{R}}_{\sum_{i=1}^{m} R_{i}}^{\alpha}(P) \subseteq(I) \overline{\mathbf{R}}_{\sum_{i=1}^{m} R_{i}}^{\alpha}(P)$ and $(I I) \underline{\mathbf{R}}_{\sum_{i=1}^{m} R_{i}}^{\alpha}(P) \subseteq(I I) \overline{\mathbf{R}}_{\sum_{i=1}^{\alpha} R_{i}}^{\alpha}(P)$ hold if and only if $R_{i} \in \mathbf{R}(i=1,2, \ldots, m)$ is a serial binary fuzzy relation between $U$ and $V$.

Based on these conceptions, we could clearly get the following results. We give some remarks that the Type - (I) and Type - (II) variable precision multi-granulation Pythagorean fuzzy rough set are generalizations of optimistic and pessimistic multi-granulation Pythagorean fuzzy rough set under the framework of two universes, respectively.

Remark 4.3 Let $(U, V, \mathbf{R})$ be the multiple granularity fuzzy approximate space under the framework of two universes. For a PFS $P$ of the universe $V, P=\left[\tau_{P}(y), \rho_{P}(y)\right], y \in V$ and $\alpha \in[0,1]$. If $\alpha=0$, some results are obtained:

$$
\begin{aligned}
& \text { (1) }(I) \underline{\mathbf{R}}_{\sum_{i=1}^{m} R_{i}}^{\alpha}(P)=\underline{\mathbf{R}}_{\sum_{i=1}^{m} R_{i}}^{O}(P),(I) \overline{\mathbf{R}}_{\sum_{i=1}^{m} R_{i}}^{\alpha}(P)=\overline{\mathbf{R}}_{\sum_{i=1}^{m} R_{i}}^{O}(P) ; \\
& \text { (2) (II) } \underline{\mathbf{R}}_{\sum_{i=1}^{m} R_{i}}^{\alpha}(P)=\underline{\mathbf{R}}_{\sum_{i=1}^{m} R_{i}}^{P}(P),(I I) \overline{\mathbf{R}}_{\sum_{i=1}^{m} R_{i}}^{\alpha}(P)=\overline{\mathbf{R}}_{\sum_{i=1}^{m} R_{i}}^{P}(P) .
\end{aligned}
$$

The conclusion of Remark 4.3 reflects on the process of attitude change for an individual, from risk preferring to risk averse, showing a continuous process for a given decision-making problem under the uncertain circumstance. Besides, the decision-makers can deal with various decisions problems by the proposed models. Furthermore, all the decision conclusions with the parameter $\alpha$ are limited to the results given by decision makers who have the attitudes of risk preferring or risk averse. Therefore, the multi-granulation rough Pythagorean fuzzy set models proposed in the proceeding part provide an effective tool to solve the decision problems under the uncertain circumstance.

Furthermore, we could prove that the optimistic and pessimistic multi-granulation rough Pythagorean fuzzy sets are special cases of Type-(I) and Type - (II) variable precision multi-granulation rough Pythagorean fuzzy set under the framework of two universes.

According to the above definitions proposed in the previous introductions, some concrete models could be obtained.

Remark 4.4 Let $(U, V, \mathbf{R})$ be the multiple granularity fuzzy approximate space under the framework of two universes. For a Pythagorean fuzzy set $P$ of universe $V$, 
$P=\left[\tau_{P}(y), \rho_{P}(y)\right], y \in V$ and $\alpha \in[0,1]$. If $R_{1}=R_{2}=\cdots=R_{m}$, for any $x \in U$ and $y \in V$, then there are some interesting results as follows:

$$
\begin{aligned}
& \mu_{\underline{R_{i}(P)}}^{\alpha}(x)=\bigwedge_{i=1}^{m}\left[\bigwedge_{\tau_{P}(y) \leq \alpha}\left(1-R_{i}(x, y) \vee \alpha\right) \wedge \bigwedge_{\tau_{P}(y)>\alpha}\left(\left(1-R_{i}(x, y)\right) \vee \tau_{P}(y)\right)\right], \\
& v_{R_{i}(P)}^{\alpha}(x)=\bigvee_{i=1}^{m}\left[\bigvee_{\rho_{P}(y) \geq(1-\alpha)}\left(R_{i}(x, y) \wedge(1-\alpha)\right) \vee \bigvee_{\rho_{P}(y)<(1-\alpha)}\left(R_{i}(x, y) \wedge \rho_{P}(y)\right)\right], \\
& \mu_{\overline{R_{i}(P)}}^{\alpha}(x)=\bigvee_{i=1}^{m}\left[\bigvee_{\tau_{P}(y) \geq(1-\alpha)}\left(R_{i}(x, y) \wedge(1-\alpha)\right) \vee \bigvee_{\tau_{P}(y)<(1-\alpha)}\left(R_{i}(x, y) \wedge \tau_{P}(y)\right)\right], \\
& v_{\overline{R_{i}}(P)}^{\alpha}(x)=\bigwedge_{i=1}^{m}\left[\bigwedge_{\rho_{P}(y) \leq \alpha}\left(1-R_{i}(x, y) \vee \alpha\right) \wedge \bigwedge_{\rho_{P}(y)>\alpha}\left(\left(1-R_{i}(x, y)\right) \vee \rho_{P}(y)\right)\right] .
\end{aligned}
$$

That is, $R_{i}(P)_{\alpha}=\left[\mu_{R_{i}(P)}^{\alpha}(x), v_{R_{i}(P)}^{\alpha}(x)\right]$ and $\overline{R_{i}}(P)_{\alpha}=\left[\mu_{\overline{R_{i}}(P)}^{\alpha}(x), v_{\bar{R}_{i}(P)}^{\alpha}(x)\right]$.

This is the variable precision multi-granulation rough Pythagorean fuzzy set model under the framework of two universes.

Next, we will discuss that both Type - (I) and Type - (II) variable precision multi-granulation rough Pythagorean fuzzy set models under the framework of two universes when there exist only a single granularity among the universes.

Remark 4.5 Let $(U, V, \mathbf{R})$ be the multiple granularity fuzzy approximate space under the framework of two universes. For a Pythagorean fuzzy set $P$ of universe $V$, $P=\left[\tau_{P}(y), \rho_{P}(y)\right], y \in V$ and $\alpha \in[0,1]$. If $R_{1}=R_{2}=\cdots=R_{m}$ and $\left(\tau_{P}(y)\right)^{2}=1-\left(\rho_{P}(y)\right)^{2}$, for any $x \in U$ and $y \in V$, some results could be obtained:

$$
\begin{aligned}
\mu_{\underline{R_{i}(P)}}^{\alpha}(x) & =v_{\underline{R_{i}(P)}}^{\alpha}(x) \\
& =\bigwedge_{i=1}^{m}\left[\bigwedge_{\tau_{P}(y) \leq \alpha}\left(1-R_{i}(x, y) \vee \alpha\right) \wedge \bigwedge_{\tau_{P}(y)>\alpha}\left(\left(1-R_{i}(x, y)\right) \vee \tau_{P}(y)\right)\right] \\
\mu_{\overline{R_{i}}(P)}^{\alpha}(x) & =v_{\overline{R_{i}}(P)}^{\alpha}(x) \\
& =\bigvee_{i=1}^{m}\left[\bigvee_{\tau_{P}(y) \geq(1-\alpha)}\left(R_{i}(x, y) \wedge(1-\alpha)\right) \vee \bigvee_{\tau_{P}(y)<(1-\alpha)}\left(R_{i}(x, y) \wedge \tau_{P}(y)\right)\right]
\end{aligned}
$$

This is $\underline{R_{i}}(P)_{\alpha}=\mu_{R_{i}(P)}^{\alpha}(x)$ and $\overline{R_{i}}(P)_{\alpha}=\mu_{\overline{R_{i}}(P)}^{\alpha}(x)$.

Besides, the two variable precision multi-granulation rough Pythagorean fuzzy set will change into the existing variable precision fuzzy rough set. 


\section{An approach to multiple attribute group decision making based on multi-granulation rough Pythagorean fuzzy set over two universes}

In the following part, a general framework of this model is proposed by the group decision making based on multi-granulation Pythagorean fuzzy rough set under the framework of two universes. Firstly, we give a reliable description of a MCGDM model on the multiple granularity approximate space under the framework of two universes. Besides, a general method to deal with the problem of MCGDM is established by the model of multi-granulation Pythagorean fuzzy rough set under the framework of two universes.

\subsection{The problem statement}

Let $U=\left\{y_{1}, y_{2}, \ldots, y_{n}\right\}$ be the decision set and $V=\left\{x_{1}, x_{2}, \ldots, x_{m}\right\}$ be the criteria set, which $x_{i}(i=1,2, \ldots, m)$ denotes evaluation criteria (i.e. style, price, location) and $y_{i}(i=1,2, \ldots, m)$ denotes decision objects (i.e. disease). $R_{1}, R_{2}, \ldots, R_{k}$ are $k$ decision makers in the group. Similar to the traditional group decision problem, individuals provide their evaluation value for all criteria $x_{i}\left(x_{i} \in V\right)$ in regards as decision set elements $y_{i}\left(y_{i} \in U\right)$. There are $R_{1}, R_{2}, \ldots, R_{k} \in F(U \times V)$, which is the evaluations of the alternative $y_{i}$ with the criteria $x_{i}$ given by the expert $k$ based on his or her experience and knowledge. $P$ is the evaluation value who has some criteria on the universe $V$ for the alternative $U$. That is, $P=\left[\tau_{P}(y), \rho_{P}(y)\right]$ for any $y \in V$ is a Pythagorean fuzzy set of the universe $V$. For a decision maker, the problem is how to make a reasonable decision on what kind of the alternative $y_{i}\left(y_{j} \in V\right)$ for the individuals should be chosen.

Therefore, an approach for MCGDM is given by using the theory of multi-granulation Pythagorean fuzzy rough set under the framework of two universes. The main method is to obtain the ranking function for the pressure of six groups in regards as the Pythagorean fuzzy set $P$ on the universe $V$ based on the multi-granulation lower approximate set $\underline{\mathbf{R}}_{\sum_{i=1}^{m} R_{i}}^{O}(P)$ and multi-granulation upper approximate set $\overline{\mathbf{R}}_{\sum_{i=1}^{m} R_{i}}^{O}(P)$. Then, we assess the pressure based on the ranking function of six groups considered.

In fact, three types of Pythagorean fuzzy rough set could be used to discuss the decision problem. In the following, we discuss the group decision making problems by using the model of optimistic multi-granulation rough Pythagorean fuzzy set under the framework of two universes.

\subsection{The model and methodology}

In the following part, we propose a methodology based on optimistic multi-granulation Pythagorean fuzzy rough set under the framework of two universes to solve the decision problems with two stages, including solving the weights of attributes and the process of information fusion.

In the first stage, firstly, the previous data should be generalized and the key attributes could be obtained by the attribute reduction based on neighbor rough set. The grey relational analysis is utilized to find the relation between the attributes and objects and considers the non-correlation degree based on the classical grey relational analysis. We utilize the 
correlation degree and non-correlation degree as the membership degree and non-membership degree of the Pythagorean fuzzy set.

In the second stage, the main goal is obtaining the final score for various groups by the multi-granulation fuzzy decision information systems under the framework of two universes established with regard to the group decision-making problem described in Sect. 5.1.

Derived from the introduction of the GDM problems in Sect. 5.1, the judgment of every individual provides a binary fuzzy relationship between the alternative set and criteria set. Besides, there is a family of binary fuzzy relations $\mathbf{R}$ between $U$ and $V$ given by decision makers, i.e., $R_{l} \in \mathbf{R}, l=1,2, \ldots, k$. As a result, we could get the multi-granulation fuzzy decision information systems under the framework of two universes $(U, V, \mathbf{R})$ for the MCGDM model given in Sect. 5.1.

Then, the optimistic multi-granulation rough lower approximate set $\underline{\mathbf{R}}_{\sum_{i=1}^{m} R_{i}}^{O}(P)$ and optimistic multi-granulation rough upper approximate set $\overline{\mathbf{R}}_{\sum_{i=1}^{m} R_{i}}^{O}(P)$ for any given decision object $P$ is calculated in regards as multiple granular fuzzy decision information systems under the framework of two universes $(U, V, \mathbf{R})$. We utilize the score function to calculate the results as is shown in Sect. 2 by Definition 2.4.

Definition 5.1 Both $\underline{\mathbf{R}}_{\sum_{i=1}^{m} R_{i}}^{O}(P)$ and $\overline{\mathbf{R}}_{\sum_{i=1}^{m} R_{i}}^{O}(P)$ are Pythagorean fuzzy sets of decision set $U$. Then, the ranking function could be obtained in regards as decision set. Denote

$$
\sum_{l=1}^{k} R_{l}(A)=\lambda \underline{R}_{\sum_{i=1}^{m} R_{i}}^{O}(P)+(1-\lambda) \bar{R}_{\sum_{i=1}^{m} R_{i}}^{O}(P) \quad \lambda \in[0,1]
$$

as the comparison law with a right weight $\lambda$ and let $\lambda$ be the optimistic parameter.

Finally, we give the ranking function according to the partial order among all decision objects determined by the priority relation $\geq$ to select the optimal alternatives.

Practically, the parameter $\lambda$ reflects on the risk attitudes of decision makers during the process of decision making. The risk attitude of decision maker becomes risk-preferring as the parameter $\lambda$ increase, and the risk attitude of decision maker becomes risk-averse as the parameter $\lambda$ decrease. As a result, the value of $\lambda$ is given by the decision maker or the empirical studies before we prepare to make decisions.

Therefore, we conduct a model to MCGDM by using the theory of multi-granulation Pythagorean fuzzy rough set under the framework of two universes. In the following, we give an example to verify the effectiveness of the proposed method.

\subsection{The algorithm}

In this subsection, we propose the algorithm of the established approach as Fig. 1. The algorithm of Process 2 is shown in the Algorithm 2. 


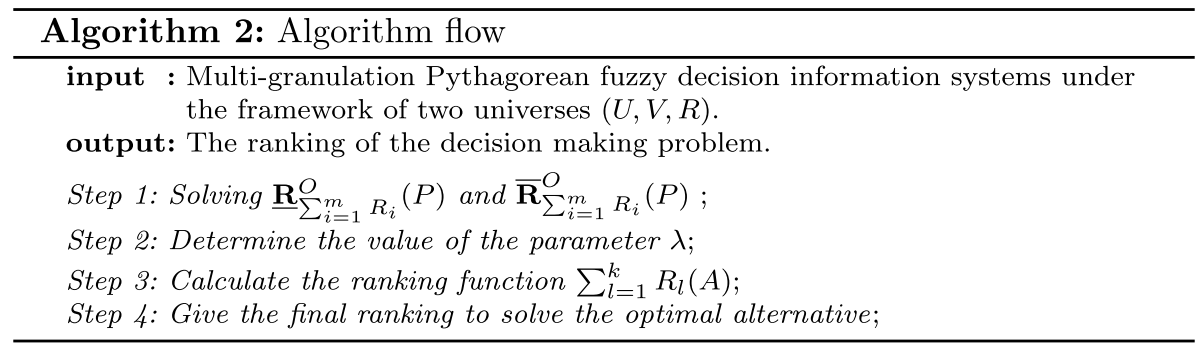

Process 1 Solving the weight of attrbutes
Process 2 The process of information fusion
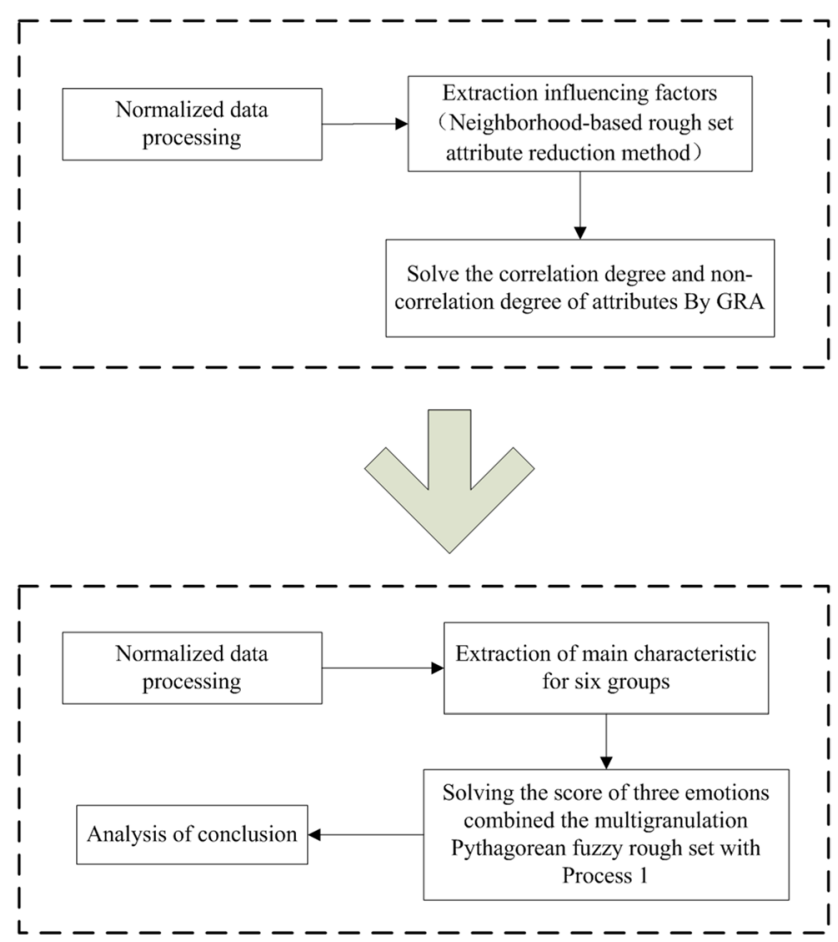

Fig. 1 The flow of the decision-making

\subsection{A case study}

By the pre-questionnaire research, we could obtain the previous data. In order to analysis the mental state of health care workers, the workers are divided into six groups, including nurses without exposure to COVID-19 as $y_{1}$, nurses in indirect contact with COVID-19 as $y_{2}$, nurses in direct contact with COVID-19 patients as $y_{3}$, physicians without exposure to COVID-19 as $y_{4}$, physicians in indirect contact with COVID-19 as $y_{5}$ and physicians in direct contact with COVID-19 patients (such as respiratory physicians) as $y_{6}$. Let $U=\left\{y_{1}, y_{2}, \ldots, y_{6}\right\}$ be the six groups and $V=\left\{x_{1}, x_{2}, \ldots, x_{19}\right\}$ be the criteria set. For the problem of psychological assessment of health care of workers in COVID-19, we choose 
Table 1 The correlation degree and non-correlation degree of complaining emotion

\begin{tabular}{llllllllll}
\hline & $x_{1}$ & $x_{3}$ & $x_{6}$ & $x_{8}$ & $x_{9}$ & $x_{11}$ & $x_{13}$ & $x_{17}$ & $x_{19}$ \\
\hline$\gamma$ & 0.7165 & 0.6095 & 0.6492 & 0.5540 & 0.6951 & 0.6805 & 0.7439 & 0.5778 & 0.4783 \\
$\rho$ & 0.2835 & 0.3905 & 0.3508 & 0.4460 & 0.3049 & 0.3195 & 0.2561 & 0.4222 & 0.5217 \\
\hline
\end{tabular}

Table 2 The correlation degree and non-correlation degree of anxiety emotion

\begin{tabular}{llllllllll}
\hline & $x_{1}$ & $x_{2}$ & $x_{3}$ & $x_{7}$ & $x_{8}$ & $x_{11}$ & $x_{13}$ & $x_{17}$ & $x_{19}$ \\
\hline$\gamma$ & 0.7164 & 0.7737 & 0.6658 & 0.5540 & 0.7297 & 0.7860 & 0.7180 & 0.7356 & 0.6140 \\
$\rho$ & 0.2836 & 0.3514 & 0.2263 & 0.3342 & 0.2703 & 0.2140 & 0.2820 & 0.2644 & 0.3860 \\
\hline
\end{tabular}

19 actions which could be influenced for the psychological state of health care workers as follows.

$x_{1}$ : Estimation of the epidemic status of the region in which it is located;

$x_{2}$ : Daily attention to COVID-19;

$x_{3}$ : The degree of risk to one's health from the epidemic;

$x_{4}$ : The degree of negative impact for the epidemic on life;

$x_{5}$ : The degree of negative impact for the epidemic on work and study;

$x_{6}$ : The possibility of being infected by the COVID-19;

$x_{7}$ : Normality of epidemic prevention measures in the region;

$x_{8}$ : When you have symptoms similar to those of COVID-19, you worry about the possibility of developing the disease;

$x_{9}$ : Refresh the latest information on the COVID-19;

$x_{10}$ : Indifferent to anything other than the COVID-19;

$x_{11}$ : Fear of the development and prevention and control for the COVID-19;

$x_{12}$ : Organize the work and study normally;

$x_{13}$ : Repeated hand washing;

$x_{14}$ : Often believe the gossip;

$x_{15}$ : Fear of meeting people who do not wear masks;

$x_{16}$ : There are always scenarios in your mind where you are infected or the COVID19 is uncontrollable;

$x_{17}$ : When someone around you doesn't care about outbreak prevention and control, you are angry and go to persuade;

$x_{18}$ : If the prevention and control work needs, willing to do some voluntary work in the vicinity of the local government and the community under the unified organization of what can be done;

$x_{19}$ : Know the symptoms of COVID-19 and measures to prevent virus infection.

We study 1054 questionnaires for the health worker, including doctors and nurses. By the attribute reduction based on the neighborhood rough set, many actions have influenced three emotions, including complaining, anxiety and confidence in overcoming the epidemic. The emotion of complaining is influenced by the actions $X_{1}=\left\{x_{1}, x_{3}, x_{6}, x_{8}, x_{9}, x_{11}, x_{13}, x_{17}, x_{19}\right\}$. The emotion of anxiety is influenced by the actions $X_{2}=\left\{x_{1}, x_{2}, x_{3}, x_{7}, x_{8}, x_{11}, x_{13}, x_{17}, x_{19}\right\}$. The emotion of confidence is influenced 
Table 3 The correlation degree and non-correlation degree of confidence emotion

\begin{tabular}{llllllllll}
\hline & $x_{6}$ & $x_{7}$ & $x_{10}$ & $x_{11}$ & $x_{12}$ & $x_{13}$ & $x_{15}$ & $x_{17}$ & $x_{18}$ \\
\hline$\gamma$ & 0.6018 & 0.7887 & 0.4864 & 0.6043 & 0.4907 & 0.5381 & 0.6379 & 0.7055 & 0.8324 \\
$\rho$ & 0.3982 & 0.2113 & 0.5136 & 0.3957 & 0.5093 & 0.4619 & 0.3621 & 0.2945 & 0.1676 \\
\hline
\end{tabular}

by the actions $X_{3}=\left\{x_{6}, x_{7}, x_{10}, x_{11}, x_{12}, x_{13}, x_{15}, x_{17}, x_{19}\right\}$. Based on the grey relational analysis, the weight of attributes could be obtained as follows (Tables 1, 2, 3):

Then, we utilize the arithmetic mean value to obtain the characteristic of the six groups. The matrix for fuzzy relation of three types of emotions could be obtained as follows:

$$
\begin{aligned}
D_{1} & =\left[\begin{array}{llllllllll}
0.0349 & 0.8889 & 0.7308 & 0.9615 & 0.1298 & 0.5714 & 0.2681 & 0.9432 & 0.0047 \\
0.1667 & 0.5000 & 0.5000 & 0.5000 & 0.2500 & 0.6667 & 0.5714 & 0.5000 & 0.2000 \\
0.4444 & 0.8000 & 0.8889 & 0.9000 & 0.1053 & 0.1250 & 0.1333 & 0.7778 & 0.2368 \\
0.0328 & 0.5000 & 0.6667 & 0.8182 & 0.0917 & 0.1250 & 0.0426 & 0.7273 & 0.0100 \\
0.2857 & 0.6000 & 0.4000 & 0.6000 & 0.2727 & 0.2500 & 0.1111 & 0.8000 & 0.0625 \\
0.7500 & 0.8333 & 0.2857 & 0.7500 & 0.1667 & 0.5000 & 0.1111 & 0.8000 & 0.2800
\end{array}\right] \\
D_{2} & =\left[\begin{array}{llllllllll}
0.0349 & 0.9718 & 0.8889 & 0.9846 & 0.9615 & 0.5714 & 0.2681 & 0.9432 & 0.0047 \\
0.1667 & 0.8889 & 0.5000 & 0.5000 & 0.5000 & 0.6667 & 0.5714 & 0.5000 & 0.2000 \\
0.4444 & 0.9630 & 0.8000 & 0.4167 & 0.9000 & 0.1250 & 0.1333 & 0.7778 & 0.2368 \\
0.0328 & 0.9897 & 0.5000 & 0.9615 & 0.8182 & 0.1250 & 0.0426 & 0.7273 & 0.0100 \\
0.2857 & 0.7368 & 0.6000 & 0.4000 & 0.6000 & 0.2500 & 0.1111 & 0.8000 & 0.0625 \\
0.7500 & 0.9474 & 0.8333 & 0.5000 & 0.7500 & 0.5000 & 0.1111 & 0.8000 & 0.2800
\end{array}\right] \\
D_{3}= & {\left[\begin{array}{lllllllll}
0.7308 & 0.9846 & 0.0787 & 0.5714 & 0.0612 & 0.2681 & 0.8214 & 0.9432 & 0.0046 \\
0.5000 & 0.5000 & 0.5000 & 0.6667 & 0.1667 & 0.5714 & 0.5000 & 0.5000 & 0.1250 \\
0.8889 & 0.4167 & 0.1176 & 0.1250 & 0.0769 & 0.1333 & 0.7500 & 0.7778 & 0.0345 \\
0.6667 & 0.9615 & 0.0093 & 0.1250 & 0.0256 & 0.0426 & 0.3846 & 0.7273 & 0.0455 \\
0.4000 & 0.4000 & 0.0833 & 0.2500 & 0.2857 & 0.1111 & 0.3333 & 0.8000 & 0.1250 \\
0.2857 & 0.5000 & 0.1250 & 0.5000 & 0.2000 & 0.1111 & 0.8000 & 0.8000 & 0.0833
\end{array}\right] }
\end{aligned}
$$

According to the multi-granulation Pythagorean fuzzy rough set proposed in Sect. 4, we utilize the optimistic multi-granulation Pythagorean fuzzy rough set to solve and analysis the problems by the fuzzy binary relation and fuzzy evaluation matrix. After the information fusion, the final results could be obtained based on the score functions for $\lambda=0.4$ and the other three approaches calculate the weight information utilizing the score function, which could transfer the Pythagorean information into the crisp number to obtain the final results by Definition 2.3 as is shown in Tables 4, 5 and 6 .

Table 4 The comparison results of complain emotion using four approaches

\begin{tabular}{ll}
\hline & The ranking results \\
\hline The original information & $y_{2}>y_{6}>y_{5}>y_{3}>y_{1}>y_{4}$ \\
The proposed method & $y_{5}>y_{1}>y_{3}>y_{2}>y_{4}>y_{6}$ \\
TOPSIS & $y_{4}>y_{5}>y_{2}>y_{3}>y_{1}>y_{6}$ \\
VIKOR & $y_{4}>y_{6}>y_{3}>y_{5}>y_{2}>y_{1}$ \\
PROMETHEE & $y_{4}>y_{5}>y_{2}>y_{3}>y_{6}>y_{1}$ \\
\hline
\end{tabular}


Table 5 The comparison results of anxiety emotion using four approaches

Table 6 The comparison results of confident emotion using four approaches

Fig. 2 The results of correlation between the MCDM method and the original evaluation based on the Euclidean distance formula

\begin{tabular}{ll}
\hline & The ranking results \\
\hline The original information & $y_{3}>y_{2}>y_{5}>y_{1}>y_{6}>y_{4}$ \\
The proposed method & $y_{2}>y_{4}>y_{3}>y_{1}>y_{5}>y_{6}$ \\
TOPSIS & $y_{4}>y_{5}>y_{2}>y_{3}>y_{1}>y_{6}$ \\
VIKOR & $y_{4}>y_{3}>y_{2}>y_{6}>y_{5}>y_{1}$ \\
PROMETHEE & $y_{4}>y_{5}>y_{2}>y_{3}>y_{6}>y_{1}$ \\
\hline
\end{tabular}

\begin{tabular}{ll}
\hline & The ranking results \\
\hline The original information & $y_{5}>y_{2}>y_{6}>y_{3}>y_{4}>y_{1}$ \\
The proposed method & $y_{6}>y_{3}>y_{5}>y_{2}>y_{4}>y_{1}$ \\
TOPSIS & $y_{4}>y_{5}>y_{3}>y_{6}>y_{2}>y_{1}$ \\
VIKOR & $y_{5}>y_{3}>y_{6}>y_{1}>y_{4}>y_{2}$ \\
PROMETHEE & $y_{4}>y_{5}>y_{3}>y_{6}>y_{1}>y_{2}$ \\
\hline
\end{tabular}

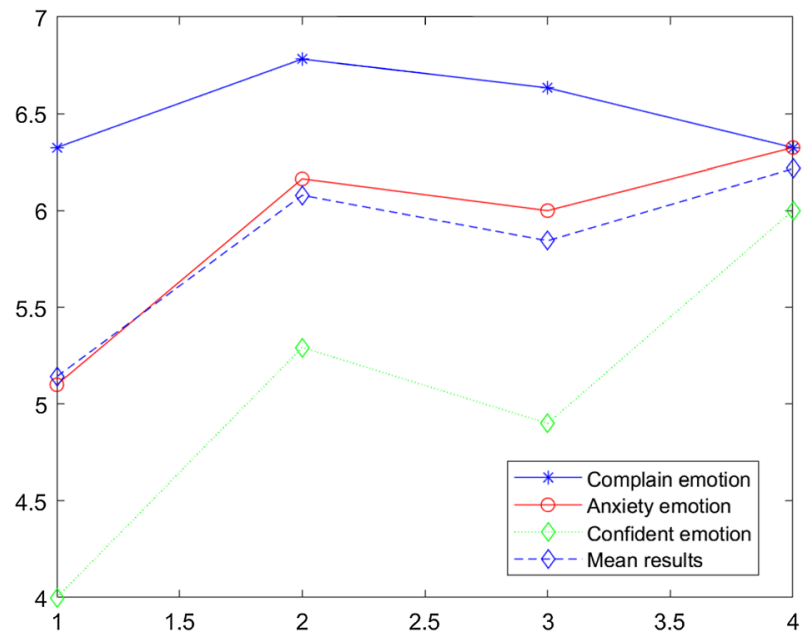

For the proposed method in this paper, we utilize the score function to solve the final score and obtain the final results. The ranking for the complain emotion is $y_{5}>y_{1}>y_{3}>y_{2}>y_{4}>y_{6}$. The ranking for anxiety emotion is $y_{2}>y_{4}>y_{3}>y_{1}>y_{5}>y_{6}$. The confident emotion is $y_{6}>y_{3}>y_{5}>y_{2}>y_{4}>y_{1}$. From the result of three emotions, the doctors without exposure to virus have the most pressure and anxiety emotion compared with other groups. The nurses in indirect contact with COVID-19 have the confident for overcoming the virus of COVID-19. Then, we compare the proposed model with other multi-criteria decision making, including TOPSIS, VIKOR and PROMETHEE (II) and the results have great differences. In order to bring the thinking patterns of machines closer to human brain perception, we compare the four approaches with the original evaluations by their own perceptions for three emotions as is shown in 
Fig. 2 by utilizing the Euclidean distance formula. From the results, the results of the proposed method have the minimum distance and are more consistent with three emotions for themselves compared with other approaches. Therefore, it shows that the results of our proposed method are more in line with individuals' own feelings and verify the validity and effectiveness.

\section{Conclusions}

Rough set theory over two universes is a generalization of Pawlak rough set. This paper studies multi-granulation rough set over tow universes combined with Pythagorean fuzzy set and application in psychological evaluation of health care workers in COVID-19. Firstly, we proposed a grey relational analysis from the perspective of relational degree and the opposite direction. Then, we presented multi-granulation Pythagorean fuzzy rough set and discussed the properties. Finally, a multi-criteria decision making is presented based on big data and applied it to psychological evaluation. A case study in COVID-19 was discussed and compared with other approaches to verify the proposed model validity and availability by comparing other multi-criteria decision making.

Compared with the existing literatures, the new contribution of this paper could be described as follows: (1) An new model solving the weight of attribute was proposed based on grey relational analysis and Pythagorean fuzzy set. (2) We generalized the multi-granulation Pythagorean fuzzy rough set over two universes and proposed multi-granulation rough Pythagorean fuzzy set over two universes. (3) The proposed method applied to the psychological evaluation of health care workers in COVID-19 could be described as the process of decision making and.

For the further study, the previous theory and traits of MGPFRS under the framework of two universes should be discussed from the perspective of management meaning and the theory models could be extended. Furthermore, this paper only considers the problem of determine the weight information and information integration and there are many directions for the multi-criteria group decision making problems, such as consensus reaching process, two sided matching decision making problem, other approaches for information integration and ranking and etc. Finally, the approach established in this paper can be applied to other practical problems in real-life.

Acknowledgements The work was partly supported by the National Natural Science Foundation of China (72071152, 7157109081774218 and 61871141), the Xi' an Science and Technology Projects(XA2020RKXYJ-0086), the Youth Innovation Team of Shaanxi Universities(2019), The Project of Guangdong Education Department (2018GWQNCX050), China Postdoctoral Science Foundation Funded Project (2020M670046ZX), Key-Area Research and Development Program of Guangdong Province (No. 2020B1111100010).

\section{Declarations}

Conflict of interest No potential conflict of interest was reported by the authors. 


\section{References}

Ali A, Ali MI, Rehman N (2018) New types of dominance based multi-granulation rough sets and their applications in conflict analysis problems. Journal of Intelligent \& Fuzzy Systems 35(3), 3859-3871 $10.3233 /$ jifs- 18757

Bashir Z, Ali J, Rashid T (2020) Consensus-based robust decision making methods under a novel study of probabilistic uncertain linguistic information and their application in forex investment. Artificial Intelligence Review 54(3), 2091-2132. DOI: 10.1007/s10462-020-09900-y

Chen TY (2018) An outranking approach using a risk attitudinal assignment model involving pythagorean fuzzy information and its application to financial decision making. Appl Soft Comput 71:460487. https://doi.org/10.1016/j.asoc.2018.06.036

Deng J (1989) Introduction to grey system theory. J Grey Syst

Faizi S, Nawaz S, Ur-Rehman A (2020) Intuitionistic 2-tuple linguistic aggregation information based on einstein operations and their applications in group decision making. Artif Intell Rev 53(6):4625-4650

Fan B, Tsang ECC, Xu W, Chen D, Li W (2018) Attribute-oriented cognitive concept learning strategy: a multi-level method. International Journal of Machine Learning and Cybernetics 10(9), 24212437. DOI: $10.1007 / \mathrm{s} 13042-018-0879-5$

Garg H (2017) Confidence levels based pythagorean fuzzy aggregation operators and its application to decision-making process. Comput Math Org Theory 23(4):546-571. https://doi.org/10.1007/ s10588-017-9242-8

Hu Q, Yu D, Liu J, Wu C (2008a) Neighborhood rough set based heterogeneous feature subset selection. Inf Sci 178(18):3577-3594. https://doi.org/10.1016/j.ins.2008.05.024

$\mathrm{Hu}$ Q, Zhao H, Yu D (2008b) Fast reduction algorithm for symbolic and numerical attributes based on neighborhood rough sets. Pattern Recognition and Artificial Intelligence 21:732-738

Huang B, Guo Cx, Zhuang Y1, Li Hx, Zhou Xz (2014) Intuitionistic fuzzy multigranulation rough sets. Information Sciences 277:299-320. DOI: 10.1016/j.ins.2014.02.064

Huang B, Wu WZ, Yan J, Li H, Zhou X (2020) Inclusion measure-based multi-granulation decisiontheoretic rough sets in multi-scale intuitionistic fuzzy information tables. Information Sciences 507:421-448. DOI: 10.1016/j.ins.2018.08.061

Jiang H, Zhan J, Chen D (2020) Promethee ii method based on variable precision fuzzy rough sets with fuzzy neighborhoods. Artificial Intelligence Review 54(2), 1281-1319. DOI: 10.1007/ s10462-020-09878-7

Khan MSA, Abdullah S (2018) Interval-valued pythagorean fuzzy gra method for multiple-attribute decision making with incomplete weight information. Int J Intell Syst 33(8):1689-1716

Kong Q, Zhang X, Xu W, Xie S (2019) Attribute reducts of multi-granulation information system. Artificial Intelligence Review 53(2), 1353-1371. DOI: 10.1007/s10462-019-09699-3

Lang G, Miao D, Fujita H (2019) Three-way group conflict analysis based on pythagorean fuzzy set theory. IEEE Transactions on Fuzzy Systems 28(3), 447-461

Li CC, Rodriguez RM, Martinez L, Dong Y, Herrera F (2019) Consensus building with individual consistency control in group decision making. IEEE Transactions on Fuzzy Systems 27(2), 319-332. DOI: $10.1109 /$ tfuzz.2018.2856125

Li J, Aswani Kumar C, Mei C, Wang X (2017) Comparison of reduction in formal decision contexts. International Journal of Approximate Reasoning 80:100-122

Li Z, Xie N, Huang D, Zhang G (2020) A three-way decision method in a hybrid decision information system and its application in medical diagnosis. Artificial Intelligence Review 53(7), 4707-4736. DOI: 10.1007/s10462-020-09805-w

Liang M, Mi J, Feng T, Xie B (2020) A dynamic approach for updating the lower approximation in adjustable multi-granulation rough sets. Soft Computing 24(21), 15951-15966. DOI: 10.1007/ s00500-020-05323-7

Liu P, Liu W (2020) Dual generalized bonferroni mean operators based on 2-dimensional uncertain linguistic information and their applications in multi-attribute decision making. Artif Intell Rev 54(1):491-517

Ma X, Zhan J, Sun B, Alcantud JCR (2020) Novel classes of coverings based multigranulation fuzzy rough sets and corresponding applications to multiple attribute group decision-making. Artif Intell Rev 53(8):6197-6256

Pang J, Guan X, Liang J, Wang B, Song P (2020) Multi-attribute group decision-making method based on multi-granulation weights and three-way decisions. Int J Approx Reason 117:122-147

Pawlak Z (1982) Rough sets. Int J Comput Inf Sci 11(5):341-356 
Pei DW, Xu ZB (2004) Rough set models on two universes. Int J Gen Syst 33(5):569-581. https://doi. org/10.1080/0308107042000193561

Peng X, Dai J (2017) Approaches to pythagorean fuzzy stochastic multi-criteria decision making based on prospect theory and regret theory with new distance measure and score function. Int J Intell Syst 32(11):1187-1214. https://doi.org/10.1002/int.21896

Peng X, Yang Y (2015) Some results for pythagorean fuzzy sets. Int J Intell Syst 30(11):1133-1160. https://doi.org/10.1002/int.21738

Qian Y, Liang J, Dang C (2010a) Incomplete multigranulation rough set. IEEE Trans Syst Man Cybern Part A: Syst Hum 40(2):420-431

Qian Y, Liang J, Yao Y, Dang C (2010b) Mgrs: a multi-granulation rough set. Inf Sci 180(6):949-970

Qian Y, Li S, Liang J, Shi Z, Wang F (2014) Pessimistic rough set based decisions: A multigranulation fusion strategy. Inf Sci 264:196-210. https://doi.org/10.1016/j.ins.2013.12.014

Shao Y, Qi X, Gong Z (2020) A general framework for multi-granulation rough decision-making method under q-rung dual hesitant fuzzy environment. Artificial Intelligence Review 53(7), 4903-4933

Sun B, Ma W (2015) An approach to consensus measurement of linguistic preference relations in multiattribute group decision making and application. Omega 51:83-92

Sun B, Ma W, Zhao H (2016) Rough set-based conflict analysis model and method over two universes. Inf Sci 372:111-125

Sun B, Ma W, Chen X, Zhang X (2018) Multigranulation vague rough set over two universes and its application to group decision making. Soft Computing 23(18), 8927-8956. DOI: 10.1007/s00500-018-3494-1

Sun L, Wang L, Ding W, Qian Y, Xu J (2020) Neighborhood multi-granulation rough sets-based attribute reduction using lebesgue and entropy measures in incomplete neighborhood decision systems. KnowlBased Syst 192. https://doi.org/10.1016/j.knosys.2019.105373

Wang X, Wang P, Yang X, Yao Y (2021) Attribution reduction based on sequential three-way search of granularity. Int J Mach Learn Cybern. https://doi.org/10.1007/s13042-020-01244-X

Wu WZ, Mi JS, Zhang WX (2003) Generalized fuzzy rough sets. Inf Sci 151:263-282. https://doi.org/10. 1016/s0020-0255(02)00379-1

Yager RR (2004) Owa aggregation over a continuous interval argument with applications to decision making. IEEE Trans Syst Man Cybern Part B-Cybern 34(5):1952-1963. https://doi.org/10.1109/tsmcb.2004.831154

Yager RR (2014) Pythagorean membership grades in multicriteria decision making. IEEE Trans Fuzzy Syst 22(4):958-965. https://doi.org/10.1109/tfuzz.2013.2278989

Yager RR, Abbasov AM (2013) Pythagorean membership grades, complex numbers, and decision making. Int J Intell Syst 28(5):436-452. https://doi.org/10.1002/int.21584

Zadeh LA (1965) Information and control. Fuzzy sets. 8(3):338-353

Zhang W (2005) Attribute reduction theory and approach to concept lattice. Sci China Ser F 48(6). https:// doi.org/10.1360/122004-104

Zhang X (2016) Multicriteria pythagorean fuzzy decision analysis: A hierarchical qualiflex approach with the closeness index-based ranking methods. Inf Sci 330:104-124. https://doi.org/10.1016/j.ins.2015.10.012

Zhang Z, Gao Y, Li Z (2020) Consensus reaching for social network group decision making by considering leadership and bounded confidence. Knowl-Based Syst 204. https://doi.org/10.1016/j.knosys.2020. 106240

Zhang Z, Gao J, Gao Y, Yu W (2021a) Two-sided matching decision making with multi-granular hesitant fuzzy linguistic term sets and incomplete criteria weight information. Expert Syst Appl 168. https:// doi.org/10.1016/j.eswa.2020.114311

Zhang Z, Li Z, Gao Y (2021b) Consensus reaching for group decision making with multi-granular unbalanced linguistic information: A bounded confidence and minimum adjustment-based approach. Information Fusion 74:96-110

Publisher's Note Springer Nature remains neutral with regard to jurisdictional claims in published maps and institutional affiliations. 


\section{Authors and Affiliations}

\section{Bingzhen Sun ${ }^{1}\left(\mathbb{D} \cdot\right.$ Sirong Tong ${ }^{1} \cdot$ Weimin $\mathrm{Ma}^{2} \cdot$ Ting Wang $^{1} \cdot$ Chao Jiang $^{3}$}

Bingzhen Sun

bzsun@xidian.edu.cn

Sirong Tong

srtong@stu.xidian.edu.cn

1 School of Economics and Management, Xidian University, Xi'an 710071, China

2 School of Economics and Management, Tongji University, Shanghai 200092, China

3 The Third Department of Neurology, the Second Affiliated Hospital of Xi' an Medical University, Xi'an, Shaanxi, China 